\title{
Ultra-Sensitive Colorimetric Plasmonic Sensing and Microfluidics for Biofluid Diagnostics Using Nanohole Array
}

\author{
Abid Ameen, ${ }^{1,2}$ Manas Ranjan Gartia,, ${ }^{2,3}$ Austin Hsiao, ${ }^{2,4}$ Te-Wei Chang, \\ Zhida $\mathrm{Xu}^{2,3}$ and Gang Logan $\mathrm{Liu}^{2,3,4}$ \\ ${ }^{1}$ Department of Materials Science and Engineering, University of Illinois at Urbana-Champaign, Urbana, IL 61801, USA \\ ${ }^{2}$ Micro and Nano Technology Laboratory, University of Illinois at Urbana-Champaign, Urbana, IL 61801, USA \\ ${ }^{3}$ Department of Electrical and Computer Engineering, University of Illinois at Urbana-Champaign, Urbana, IL 61801, USA \\ ${ }^{4}$ Department of Bioengineering, University of Illinois at Urbana-Champaign, Urbana, IL 61801, USA
}

Correspondence should be addressed to Zhida Xu; zhidaxu1@illinois.edu and Gang Logan Liu; loganliu@illinois.edu

Received 11 November 2014; Revised 5 January 2015; Accepted 6 January 2015

Academic Editor: Antonios Kelarakis

Copyright (C) 2015 Abid Ameen et al. This is an open access article distributed under the Creative Commons Attribution License, which permits unrestricted use, distribution, and reproduction in any medium, provided the original work is properly cited.

\begin{abstract}
Colorimetric techniques provide a useful approach for sensing application because of their low cost, use of inexpensive equipment, requirement of fewer signal transduction hardware, and, above all, their simple-to-understand results. Colorimetric sensor can be used for both qualitative analyte identification as well as quantitative analysis for many application areas such as clinical diagnosis, food quality control, and environmental monitoring. A gap exists between high-end, accurate, and expensive laboratory equipment and low-cost qualitative point-of-care testing tools. Here, we present a label-free plasmonic-based colorimetric sensor fabricated on a transparent plastic substrate consisting of about one billion nanocups in an array with a subwavelength opening and decorated with metal nanoparticles on the side walls, to bridge that gap. The fabrication techniques of the plasmonic sensor, integration to portable microfluidic devices for lab on chip applications, demonstration of highly sensitive refractive-index sensing, DNA hybridization detection, and protein-protein interaction will be reviewed. Further, we anticipate that the colorimetric sensor can be applied to point-of-care diagnostics by utilizing proper surface functionalization techniques, which seems to be one of the current limiting factors. Finally, the future outlook for the colorimetric plasmonic sensors is discussed.
\end{abstract}

\section{Introduction}

Most biochemical assay or sensing methods require labels such as fluorescent, radioactive or enzymes (e.g., in enzyme-linked immunosorbent assays (ELISAs)) to detect biomolecules [1]. The label molecule is attached to the target molecule and it is the label molecule that is detected rather than the target molecule. Label-free sensing does not need any label but can directly detect the presence of target molecules [2]. Plasmonics take advantage of the coupling of light to bound charges like electrons in metals and allow breaking the diffraction limit for the localization of light into subwavelength dimensions enabling strong field enhancements [3]. The shape of the nanoparticle extinction and scattering spectra and in particular the peak wavelength, $\lambda_{\max }$, depends on the nanoparticle composition, size, shape, orientation, and local dielectric environment so it can be used for sensing applications [4]. For the surface plasmon resonance (SPR) sensor, the peak wavelength at plasmon resonance is shifted by different refractive index of the environment but complicated instrumentation such as Otto setup, Kretschmann configuration, and spectrometer is required to detect the shift of the resonance peak [5]. Early in 1983, Liedberg et al. proposed using the plasmon sensor for label-free immunoassay [6]. In recent years, different types of sensors using surface plasmon have been implemented, including the surface plasmon resonance (SPR) sensor for cell adhesion studies [7], detection of metal ion [8], and clinical diagnosis of type I allergies [9]; localized surface plasmon resonance (LSPR) of a single particle for detection of proteins [10], colorimetric visualization of concentration of glucose [11], and colorimetric enzyme-linked immunosorbent assay [12]; surface enhanced Raman scattering (SERS) sensors for detection of DNA molecule [13], water contaminants [14], 


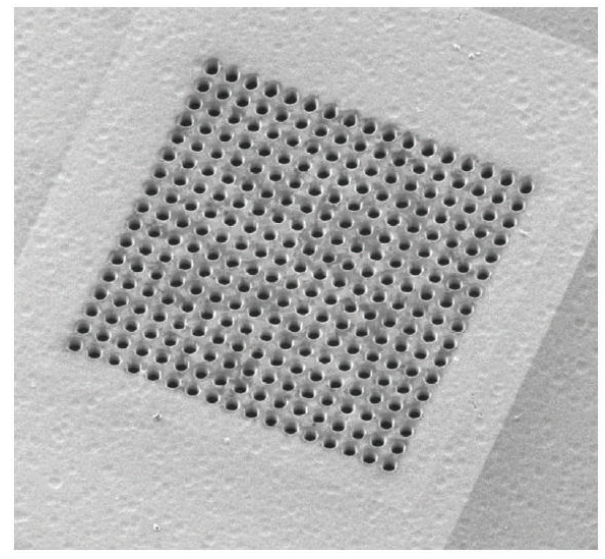

(a)

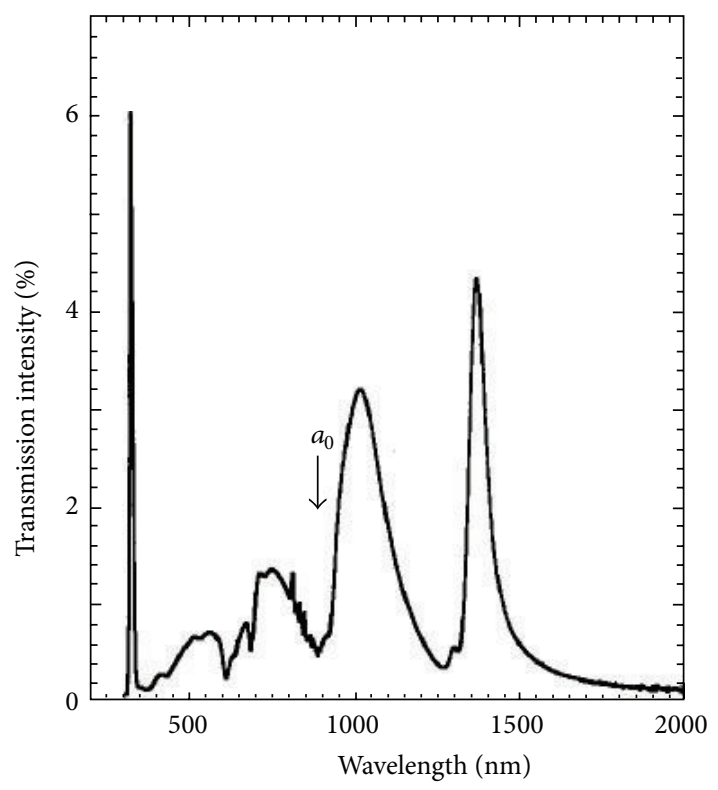

(b)

Figure 1: (a) Periodic nanohole arrays with square array of holes show EOT [28]. (b) Transmission spectrum of EOT sensor using Ag film of thickness $200 \mathrm{~nm}$, hole diameter $150 \mathrm{~nm}$, and periodicity, $a_{0}=900 \mathrm{~nm}$ [87].

melamine [15], and yeast cells [16]. Compared to chemicalbased sensors, surface plasmon-based sensors have the advantages of versatility, high sensitivity, reusability, and a label-free property.

Generally, surface plasmon sensors are divided into two categories: localized surface plasmon sensor (LSPR) and propagating surface plasmon sensor (SPR). LSPR sensors are composed of discrete nanoparticles (ordered or random) either in solution or on solid substrates. In literature, several excellent review articles exist that deals with LSPR biosensor design, their underlying physics, and application for sensing of biomolecules [4, 17-23]. Here, we will mainly focus on SPR sensors. Conventional prism coupled SPR sensors have already entered the realm of commercial market and will not be reviewed here. Instead, interested readers are directed to recent review and perspective on conventional SPR sensors $[24,25]$. Another form of SPR sensors involving extraordinary optical transmission (EOT), which is composed of periodic array of nanoholes, has been widely used for biosensing applications. Recently, nanohole sensors integrated with microfluidics using spectroscopic-based measurements have been reviewed [26]. Another recent review also deals with use of plasmonic sensors to point of care applications [27]. In this review, we will mainly focus on EOT-based nanohole sensors using intensity-based measurement systems as opposed to spectroscopic-based measurement systems.

Krishnan et al. were the first to propose extraordinary optical transmission (EOT) of light through subwavelength holes in silver films due to the excitation of surface plasmon resonance (SPR) as shown in Figure 1(a) [28]. The incident light coming from top, first couple to SPR at the top due to the periodic hole array, then the light tunnels through the hole and couple to SPR at the bottom surface of metallic film. Finally, the light is reemitted from the bottom surface with multiple resonance peaks as shown in Figure 1(b). The resonance peak position of the transmission spectrum at normal incidence can be approximately calculated using the surface plasmon dispersion relation as $[29,30]$

$\lambda_{\max }=\frac{a_{0}}{\sqrt{i^{2}+j^{2}}} \sqrt{\frac{\varepsilon_{m} \varepsilon_{d}}{\varepsilon_{m}+\varepsilon_{d}}} \quad$ (square lattice)

$\lambda_{\max }=\frac{a_{0}}{\sqrt{(4 / 3)\left(i^{2}+i j+j^{2}\right)}} \sqrt{\frac{\varepsilon_{m} \varepsilon_{d}}{\varepsilon_{m}+\varepsilon_{d}}} \quad$ (triangular lattice),

where $a_{0}$ is the period of the nanohole array, $\varepsilon_{m}$ and $\varepsilon_{d}$ are the permittivity of the metal and the dielectric material in contact with the metal, respectively, and $i$ and $j$ are the scattering orders from the hole array. As shown in (1), the resonance peak position is proportional to the periodicity of the nanohole array. Hence, sensor resonance properties can be tuned by adjusting the periodicity of the hole array. For example, the color transmitted by the sensor can be tuned from blue to red by varying the periodicity of the nanoholes from $230 \mathrm{~nm}$ to $410 \mathrm{~nm}$ as shown in Figures 2(a) and 2(c). Figure 2(b) shows an SEM image of the sensor with lattice constant of $330 \mathrm{~nm}$ and the corresponding microscopic image at the inset transmitting green light. With the increase in the distance between the holes (i.e., increasing the lattice constant $a_{0}$ ), the transmission resonance peaks are generally red shifted as shown in Figure 2(d). However, as shown in Figure 1(b), the EOT structures are associated with appearance of multiple peaks in the visible range due to coupling of resonances among holes and asymmetric 


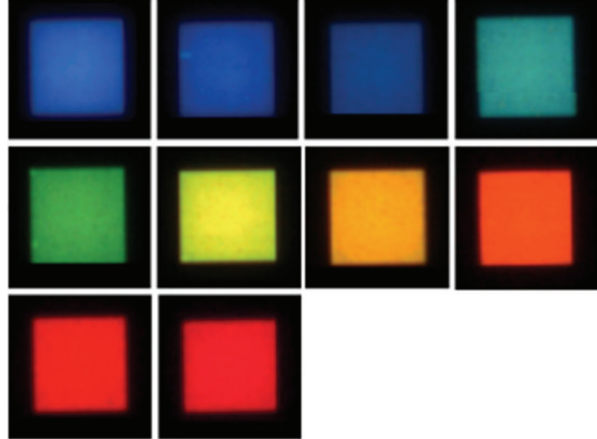

(a)

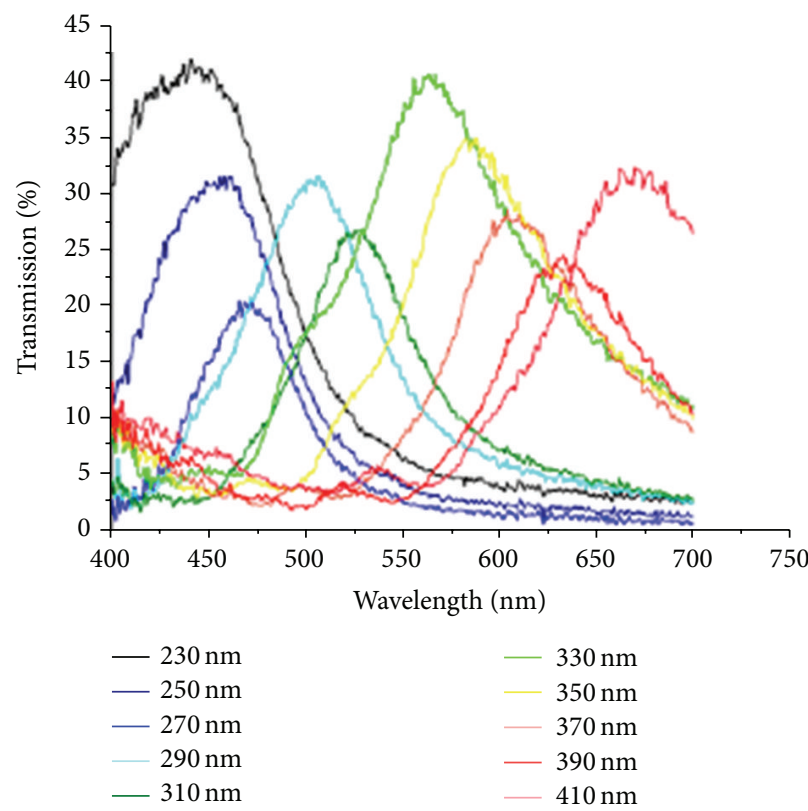

(c)

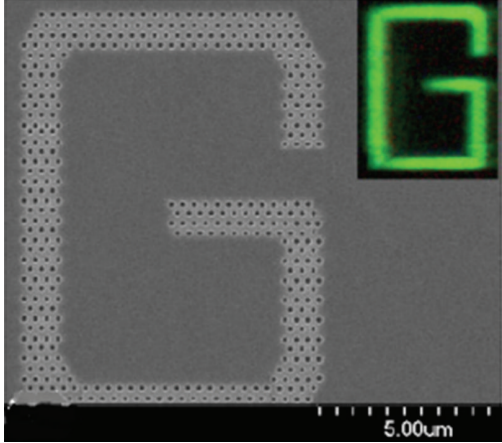

(b)

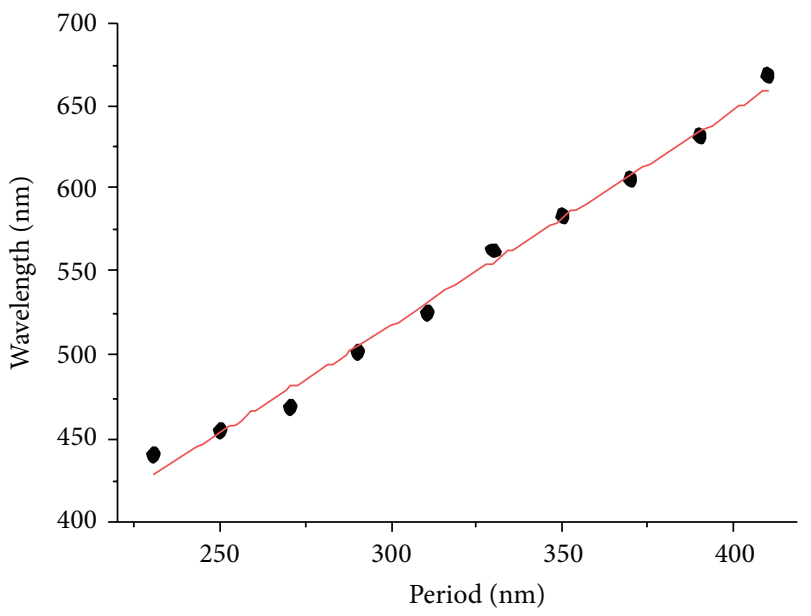

(d)

FIGURE 2: Plasmonic nanohole array transmitting different colors based on periodicity of the holes. (a) Images of various nanohole array in transmission mode under a white light illumination. (b) A SEM image of holes composing the letter "G" with periodicity of $330 \mathrm{~nm}$. The transmitted light image of the structure is shown in the inset. (c) Transmission spectra of the nanohole arrays are shown in (a). (d) Dependence of transmission peak of the nanohole array on the period of the holes. Adapted from [88].

boundary conditions (low dielectric materials like air on top and high dielectric materials like glass/quartz at the bottom of the metallic film). For colorimetric sensor it is advantageous to have single peak. There are several fabrication techniques that can be applied to making such sensor, which will be discussed next.

\section{Fabrication of Plasmonic Sensors}

Plasmonic structures, free-standing noble-metal structures, or metal deposited over nanophotonics structures has attracted attentions in recent years due to its capability to guide and manipulate light at nanoscale dimensions. Different metal nanoparticle shapes such as spheres, rods, cubes, boxes, bowls, rice, pyramids, and cages [31,32] have been achieved through chemical synthesis processes. Although solution-based methods are scalable, achieving uniformity in size and shape and controlling the space between the structures within a matrix are still a challenge [33]. Direct writing methods such as electron beam lithography (EBL) and the focused ion beam (FIB) have also been used to generate plasmonic nanostructure with lateral resolution of less than $10 \mathrm{~nm}$ [34], which is mainly limited by electron or ion scattering in the polymer matrix and swelling of the resist in the developer solution. Additional processing steps including metal deposition and pattern are required for completing the fabrication process, which further limit the resolution. The metal films using electron beam evaporation and sputtering methods are inherently polycrystalline and the rough-grain boundaries in polycrystalline noble metal surfaces introduce undesirable scattering and ohmic losses in the plasmonic devices.

Recently, Teri Odom's lab at Northwestern University has developed a new technique called PEEL (combining 


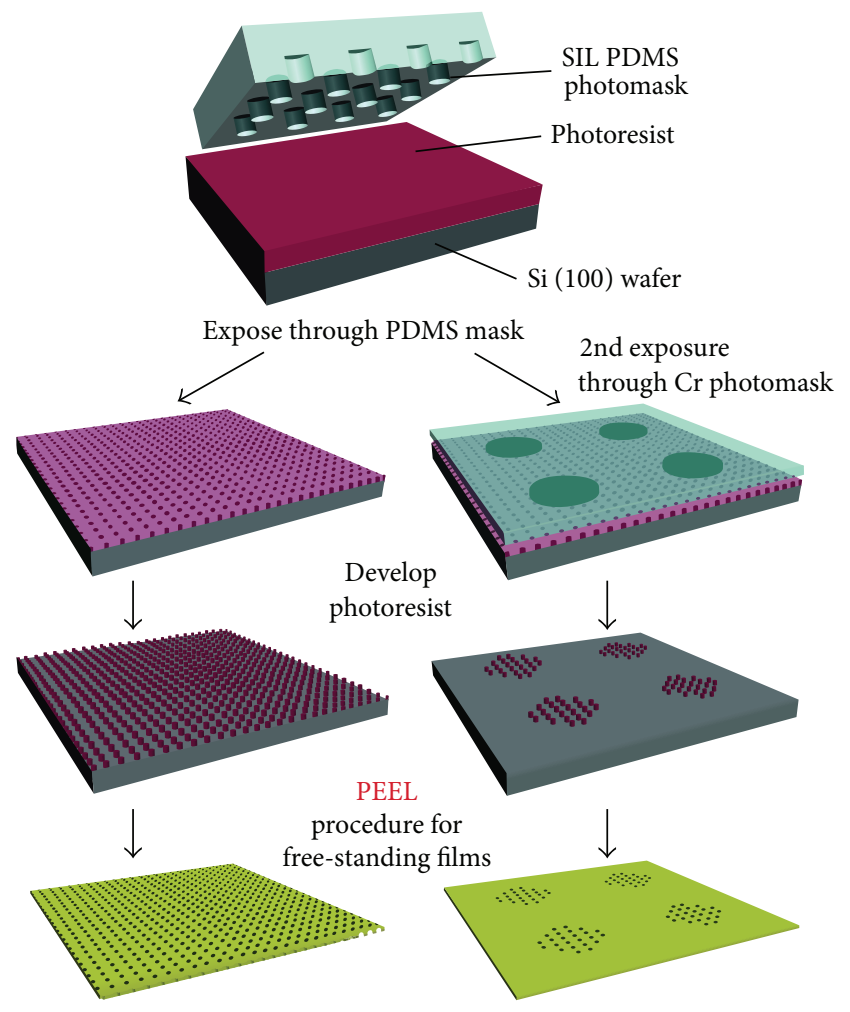

(a)
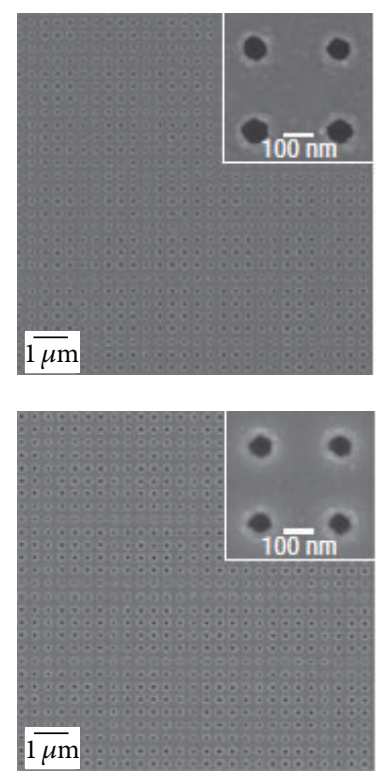

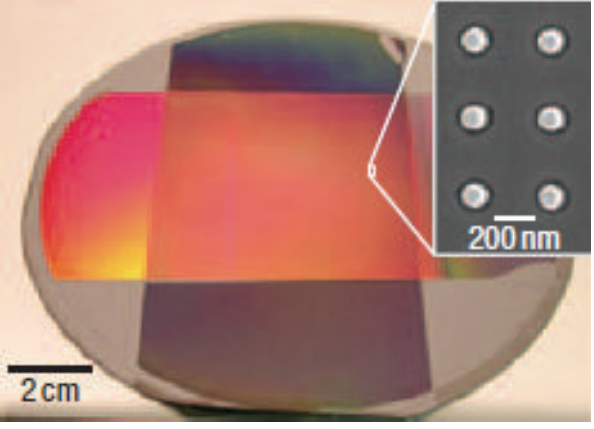

(b)
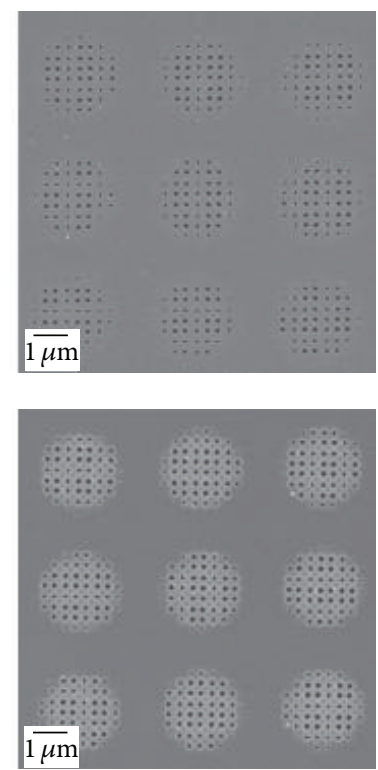

(c)

FIGURE 3: Soft interference lithography. (a) Schematic showing the PEEL (photolithography, etching, electron-beam deposition, and lift-off of the film) process. (b) Optical image of master mold used in the PEEL process. The area of the mold is about $14 \mathrm{~cm} \times 14 \mathrm{~cm}$ and it consists of array of Si post with diameter $=100 \mathrm{~nm}$, height $=400 \mathrm{~nm}$, and pitch $=400 \mathrm{~nm}$. SEM image of the post is shown in the inset. (c) SEM image of the replicated nanohole array is from the master mold. Adapted from [89].

PSP (phase-shifting photolithography), etching, electronbeam deposition, and lift-off) to transfer patterned features in a photoresist into free-standing, functional materials (Figure 3) [35]. The key attributes of using EBL and FIB to generate plasmonic nanostructures are of high resolution, controllability in design, and shape and also fewer defects $[36,37]$. However, tools like EBL, FIB, or deep-UV projection lithography are very expensive and are difficult to scale for 


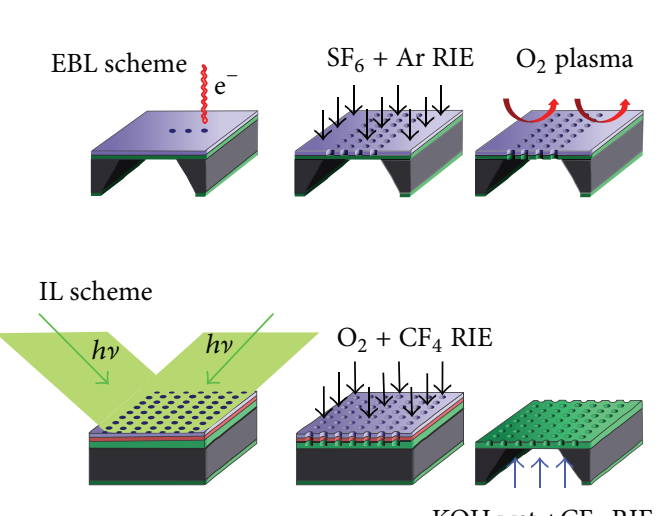

$\mathrm{KOH}$ wet $+\mathrm{CF}_{4} \mathrm{RIE}$

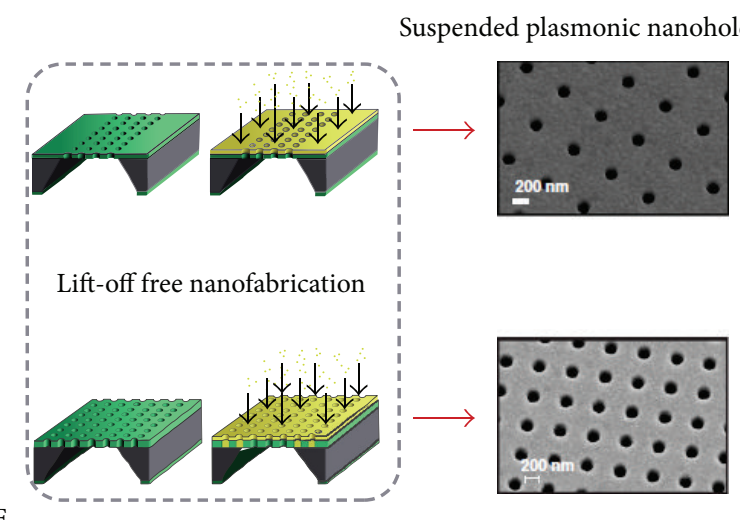

(a)

(1) Single-crystal silicon

(2) Standard patterning

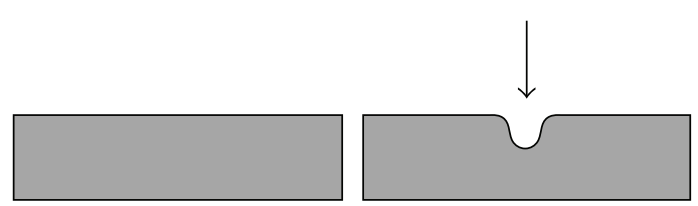

(3) Deposit metals and a backing layer

(4) Peel off to reveal

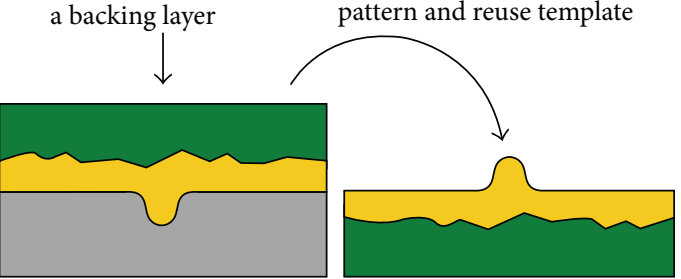

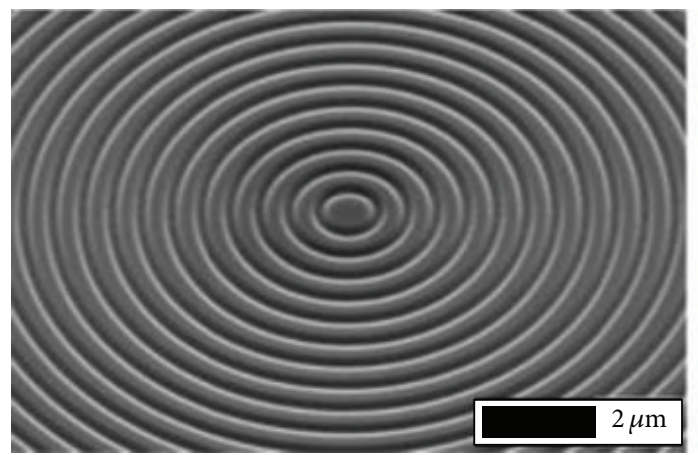

Silicon template

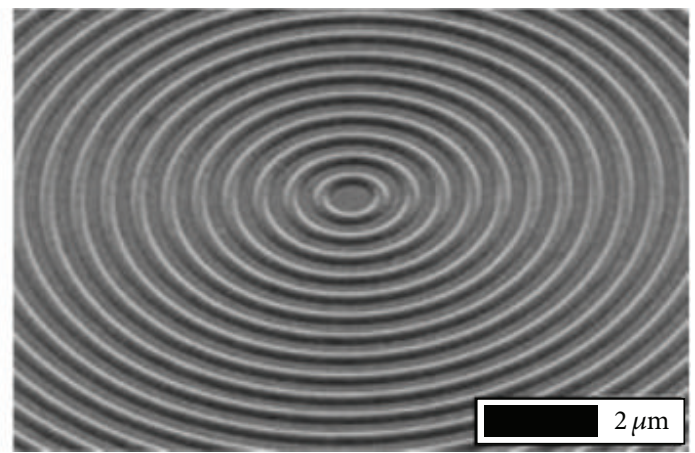

Patterned silver

(b)

FIGURE 4: (a) Lift-off free evaporation (LIFE) method of forming nanoholes. The method involves making holes on the underlying SiNx membrane instead of the PMMA resist. The backetching of Si supporting substrate enables a suspended plasmonic nanohole structure. Both electron-beam lithography (EBL) and interference lithography (IL) were used to make the nanohole structures. SEM images on the right show fabricated devices showing nanohole array with period of $600 \mathrm{~nm}$, and diameter of $180 \mathrm{~nm}$ made using EBL process. Using IL process the periodicity was $580 \mathrm{~nm}$ and the diameter of the holes was $230 \mathrm{~nm}$. Adapted from [78]. (b) Schematic of template stripping methods to make plasmonic devices with smooth surfaces. This process takes advantage of the poor adhesion and good wettability of noble metals on silicon. SEMs of the silicon template and template stripped silver device are shown at the bottom. Adapted from [90].

large-scale fabrication of plasmonic structures because of the low throughput due to the serial process.

The ablation mechanism in FIB is a complex combination of the photochemical and photothermal processes. The etch rate selection of different materials for ablation is also a challenge as the etched fragments can be redeposited on the projection lens which can damage the optics. To overcome these problems various soft lithography techniques such as replica molding, microtransfer printing, and nanoimprint lithography (NIL) [38-41] are used in which the master template is mechanically pressed into a polymer material (target substrate) to transfer the features from the master mold followed by curing of the polymer material with cooling, heating or ultraviolet (UV) light. In order to produce ultrasmooth plasmonic surfaces, a nanomanufacturing method called template stripping has been introduced as shown in Figure 4 [42]. In general, noble metals have poor adhesion but good wettability on ultrasmooth templates such as freshly cleaved mica or silicon wafers. Template stripping takes advantage of the above properties and works in such a way that, first, a metal film is deposited onto the clean template and then an epoxy or adhesive backing layer is attached to the topside of the metal film. By peeling off the supporting polymer layer, the metal will be released from the template.

Logan Liu's group has fabricated the plasmonic structure using the replica molding process where the master nanocone 

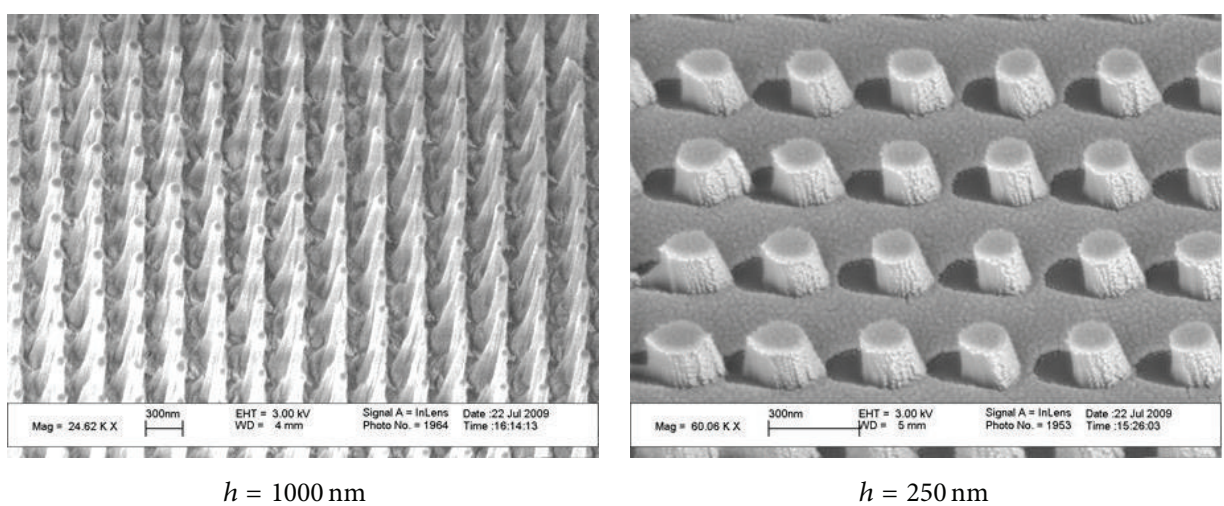

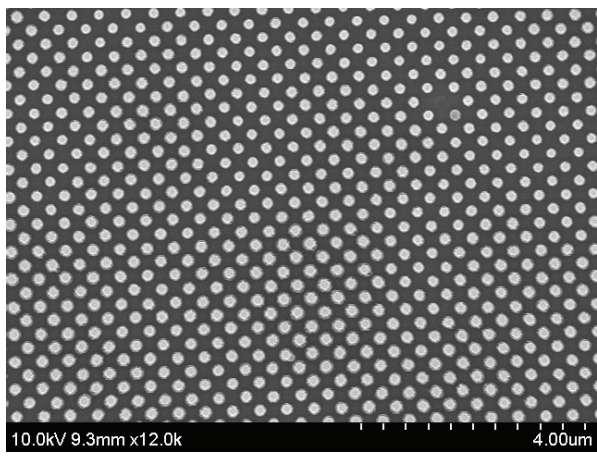

$h=500 \mathrm{~nm}($ top view)

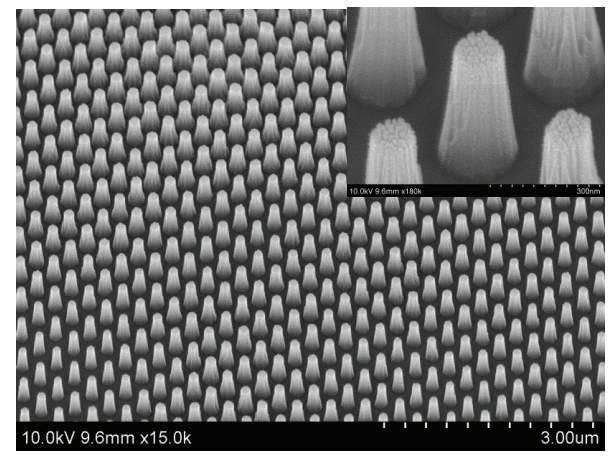

$h=500 \mathrm{~nm}\left(30^{\circ}\right.$ tilt view $)$

FIGURE 5: Scanning electron micrograph (SEM) of the master mold used for the fabrication of nanoplasmonic device. The master mold is fabricated on a glass substrate using laser interference lithography technique [91, 92] with a range of different heights; $h=1000 \mathrm{~nm}$ (top left), $h=250 \mathrm{~nm}$ (top right), $h=500 \mathrm{~nm}$ top view (bottom left), and $h=500 \mathrm{~nm} 30^{\circ}$ tilted view (bottom right) [46].

pattern is fabricated on a glass substrate using the laser interference lithography technique $[43,44]$ with a range of different heights $(h=250,500$, and $1000 \mathrm{~nm})$. Figure 5 shows the SEM images of the master mold. The two-dimensional square lattice of master mold (pitch, $p=350 \mathrm{~nm}$ ) is transferred to a flexible and optically transparent polyethylene terephthalate (PET) film using nanoreplica molding process. Figure 6 shows the schematic of the replica molding fabrication process. The nanocone master made on glass substrate is first cleaned and silanized for $30 \mathrm{~min}$ followed by ethanol and deionized water rinse (Figure 6(a)). The $5 \mu \mathrm{L}$ of UV-curable polymer (NOA-61) is evenly spread on the top of the nanocone master and a PET sheet is carefully put on top of the polymer, to avoid the bubble formation and to act as a substrate. The master with the polymer and PET sheet was then exposed to UV-light $\left(105 \mathrm{~mW} \mathrm{~cm}^{-2}\right)$ for $60 \mathrm{sec}$ (Figure 6(b)). The PET substrate with nanohole arrays was peeled off carefully from the master mold (Figure 6(c)) and then in order to make the structure surface plasmon active, $90 \mathrm{~nm}$ of metal layer of gold or silver is deposited (Figure 6(d)). From the SEM images of the plasmonic device obtained using replica a molding process is shown in Figure 7, and it is quite clear that nanoscale features can be transferred to the polymer substrate from the original master mold with high fidelity. This process, using replica molding, is used to make a low-cost, wafer-scale plasmonic device with a simple methodology and high resolution and also opens up opportunity for biosensing applications. However, due to imperfect replica molding, defects can be introduced on the plasmonic device which can broaden the resonance.

\section{Sensitivity of Plasmonic Sensor}

As shown in Table 1, the sensitivity of plasmonic sensors is limited to about $1000 \mathrm{~nm} / \mathrm{RIU}$ in the visible range. High sensitivity of more than $1500 \mathrm{~nm} / \mathrm{RIU}$ is shown in the IR and near IR region using plasmonic array sensor [45]. Recently, Gartia et al. reported a surface plasmon-based sensors with sensitivity of $46,000 \mathrm{~nm} / \mathrm{RIU}$ using cup shaped nanohole array (also known as the nano-Lycurgus cup array (nanoLCA)) [46]. NanoLCA enables detection of reactions that induce a small change of the refractive index such as DNA hybridization even by naked eye. Figure 8 presents a comparison of geometrical differences between common EOT structures and the nano-LCA structure for enhancing the plasmonics characteristics [46]. First, the tapered geometry will funnel and adiabatically focus the photons on the subwavelength plasmonic structure at the bottom, leading to large local electric field and enhancement of EOT (due to radiative coupling of surface plasmons). Secondly the localized resonance supported by $3 \mathrm{D}$ nano-LCA will enable broadband tuning of optical transmission through controlling the shape, size, and period of holes as well as the shape, size, and period of metallic particles decorated at 


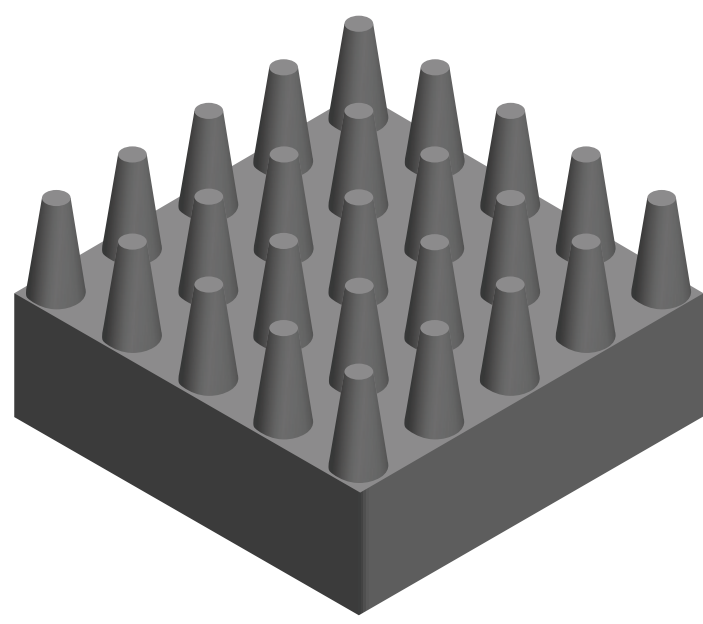

(a)

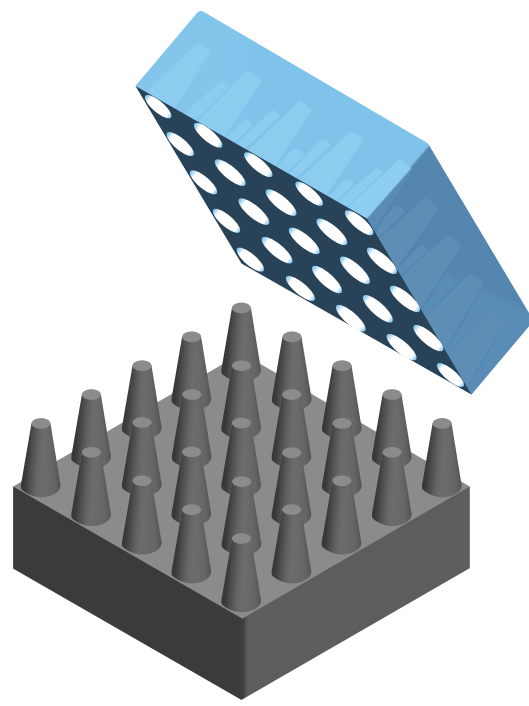

(c)

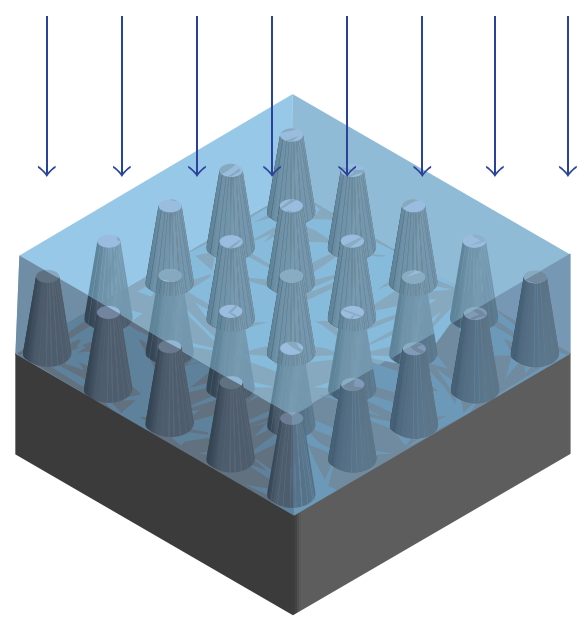

(b)

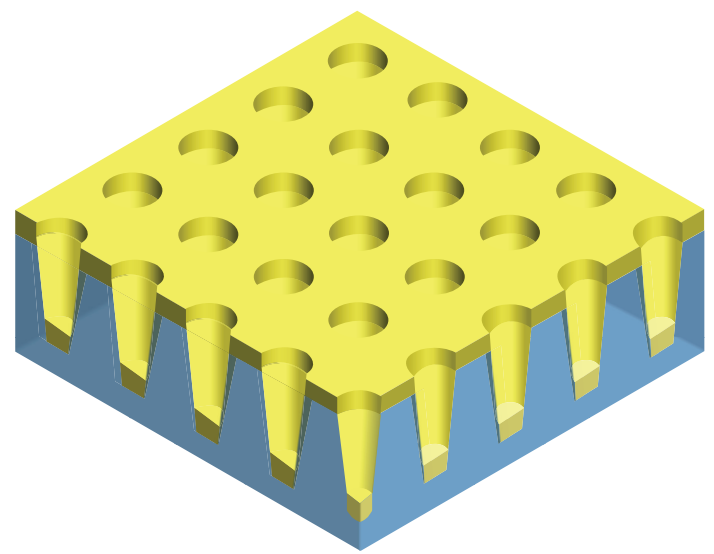

(d)

FIGURE 6: Schematic overview of the replica molding fabrication process. (a) The nanocone master mold made on glass substrate is first cleaned and silanized for $30 \mathrm{~min}$ followed by ethanol and deionized water rinse. (b) $5 \mu \mathrm{L}$ of UV-curable polymer (NOA-61) is evenly spread on the top of the nanocone master and a PET sheet is carefully, to avoid the bubble formation, put on top of the polymer to act as a substrate. The master with the polymer and PET sheet then exposed to UV-light $\left(105 \mathrm{~mW} \mathrm{~cm}^{-2}\right)$ for $60 \mathrm{sec}$. (c) The PET substrate with nanohole arrays is peeled off carefully from the master mold. (d) Then and then in order to make the structure surface plasmon active, $90 \mathrm{~nm}$ of metal layer of gold or silver is deposited [46].

the side walls. Moreover, the LSPs mediated transmissions in nano-LCAs are expected to be of higher magnitude than the SPPs mediated transmission in typical 2D hole arrays. Figure 9 shows how the nanocup array sensor changes color when different liquids are dropped on its surface. Figure 9(a) shows the white light transmitted by a hole with the shape of wave function " $\psi$." Figure 9(b) shows the same hole with the nanocup array sensor on top of it. The image is taken in transmission mode, when air $(n=1)$ is present, and the surface of nano-LCA sensor appears green. Similarly, Figure 9(c) shows an array of holes and Figure 2(d) shows the corresponding transmission image of nano-LCA on top of it. The scale bars for both the images are $100 \mu \mathrm{m}$. The dotted area in Figure 9(d) shows the color orange when sodium chloride solution partially covers the surface of the nano-LCA. Figure 9(e) shows the vibrant color produced by the nano-LCA when the different solutions (with refractive indices $n>1$ ) are dropped on the surface. The transmission spectrum will redshift and it will redshift more with larger $n$ values. In Figure 9(e), oil with larger $n$ values appears redder compared to that of the sodium chloride $(\mathrm{NaCl})$ solution.

Due to longer decay length (200-300 $\mathrm{nm}$ for SPR [47] and 5-10 $\mathrm{nm}$ for LSPR [48]) of the evanescent surface plasmon wave, SPR-based sensors have a higher sensitivity to bulk refractive index changes, whereas LSPR sensors are more sensitive to surface refractive index changes such as a molecular adsorption processes [23]. However, due to longer decay length of the evanescent wave, the results obtained from the 


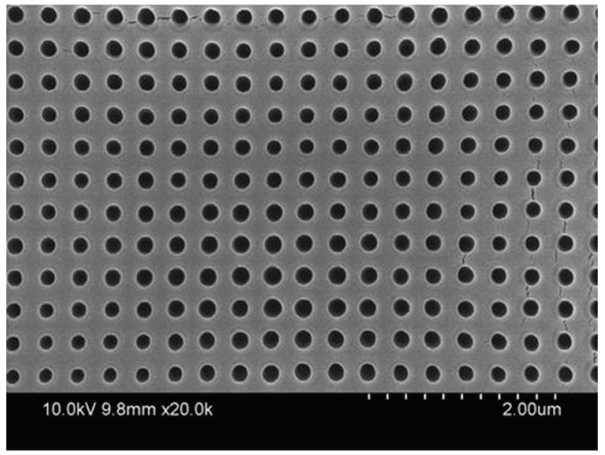

(a)

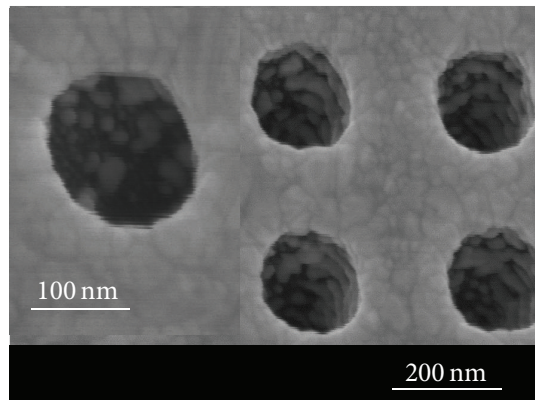

(c)

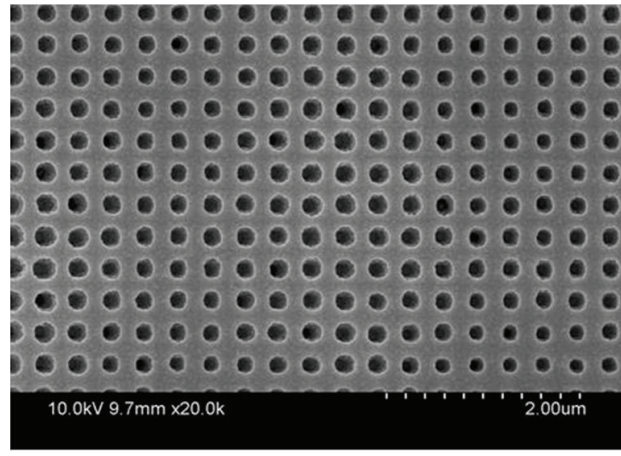

(b)

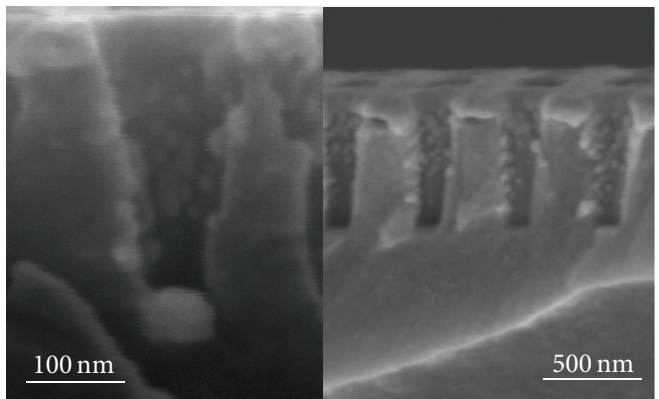

(d)

FIGURE 7: SEM image of the plasmonic device fabricated using replica molding method. (a) SEM (top view) image of polymer nanohole structure before deposition of metal. (b) SEM (top view) image of nanohole structure after $90 \mathrm{~nm}$ deposition of Ag. Top (c) and cross-sectional (d) view SEM images of plasmonic device showing the nanoparticles inside the nanohole structure [46].

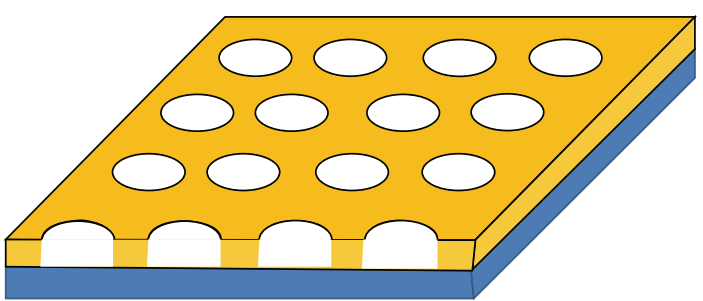

2D nanohole array

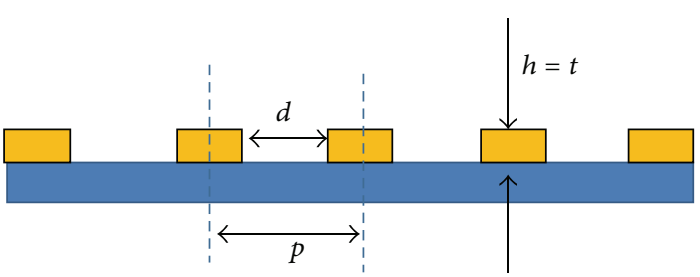

Cross-sectional view

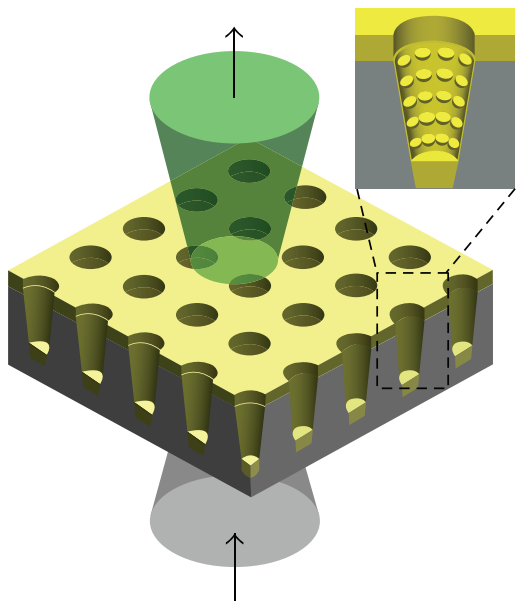

3D nano-Lycurgus cup array (nano-LCA)

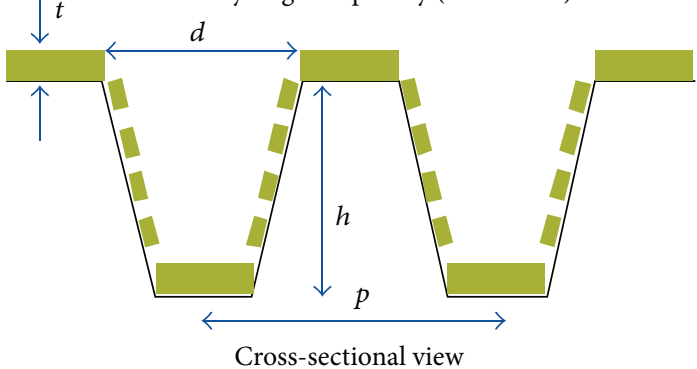

FIGURE 8: Geometrical comparison of conventional 2D nanohole plasmonic substrate versus 3D nanoLycurgus cup array (nano-LCA)-based plasmonic substrate [46]. 


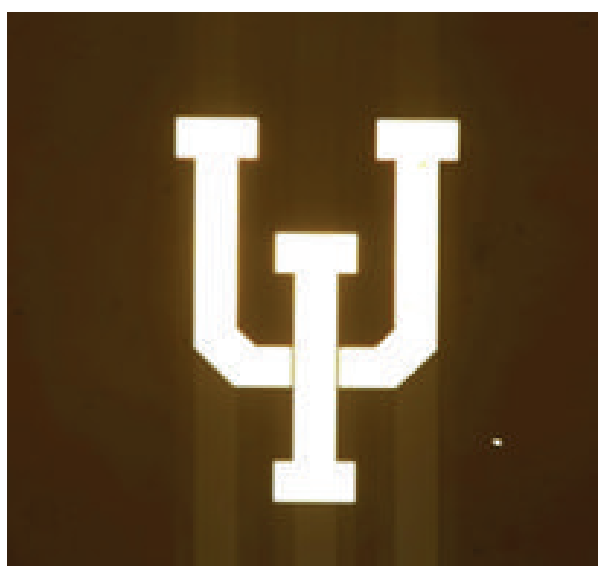

(a)

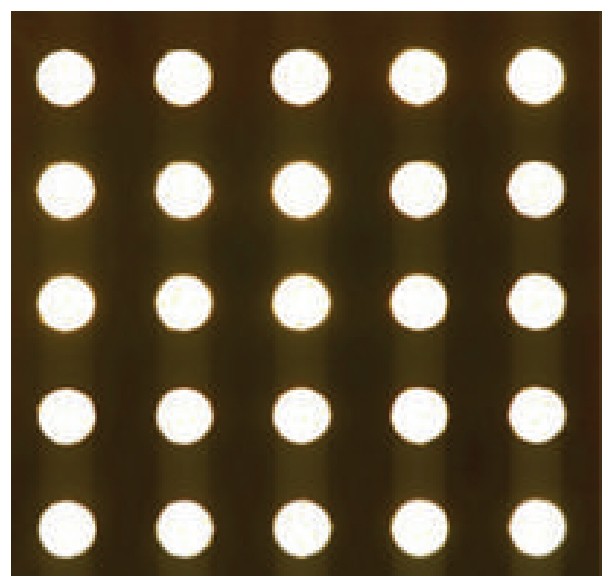

(c)

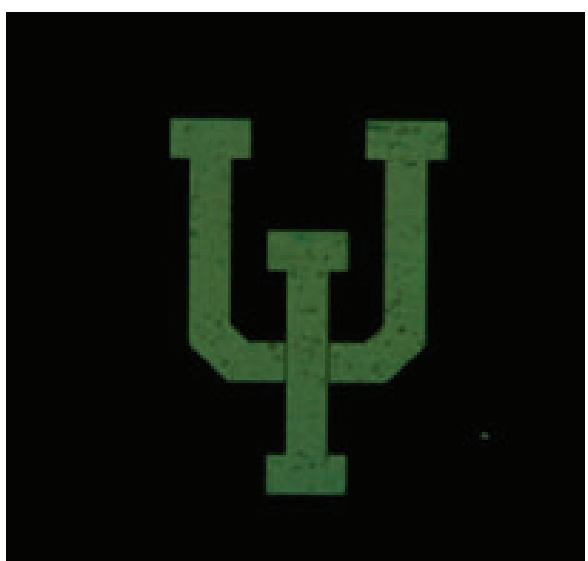

(b)

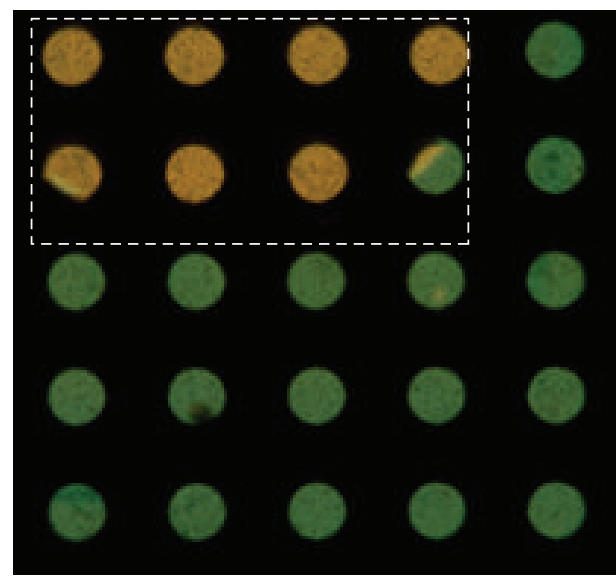

(d)

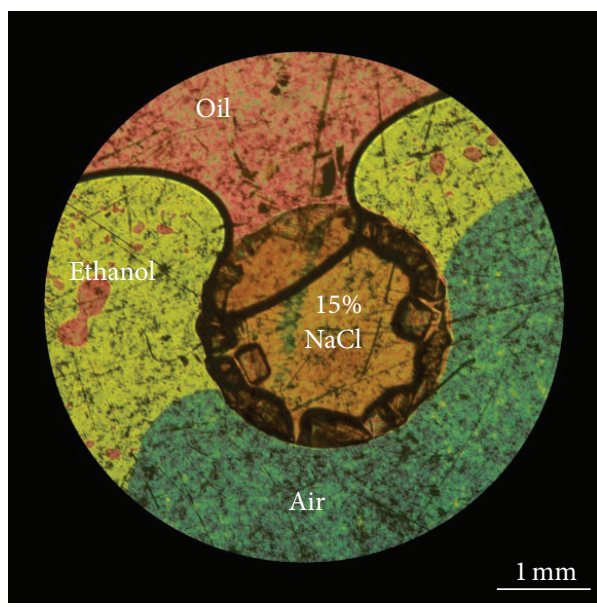

(e)

FIGURE 9: Colorimetric plasmonic sensing with nanocup array device. Bright field image ((a) and (c)) without and ((b) and (d)) with the sensor. The green color is with air $(n=1)$ and the orange color (inside the dotted area) is due to $\mathrm{NaCl}$ solution on top of the sensor. (e) Mixture of ethanol, oil, and $\mathrm{NaCl}$ shows distinctive colors on the sensor. 
TABLE 1: Comparison of sensitivity of different plasmonic-based sensors.

\begin{tabular}{|c|c|c|c|c|c|c|}
\hline Nanostructure & $\begin{array}{l}\text { Wavelength } \\
\quad(\mathrm{nm})\end{array}$ & $\begin{array}{c}\text { Structural } \\
\text { dimensions }(\mathrm{nm})\end{array}$ & Characteristics & $\begin{array}{l}\text { Sensitivity } \\
\text { (nm/RIU) }\end{array}$ & RI range & Reference \\
\hline $\begin{array}{l}\text { Gold colloidal } \\
\text { nanoparticles }\end{array}$ & $450-600$ & $d: 30$ & $\begin{array}{c}\text { Shape-dependent } \\
\text { LSPR } \\
\end{array}$ & 70.9 & $1.32-1.5$ & {$[80]$} \\
\hline $\begin{array}{l}\text { Arrays of gold } \\
\text { nanodisk }\end{array}$ & $500-1,000$ & $p: 162,340$ & $\begin{array}{c}\text { Anisotropic property } \\
\text { of gold nanodisk }\end{array}$ & $167-327$ & $1.32-1.42$ & [81] \\
\hline Gold nanorods & $800-1,000$ & $\begin{array}{l}\text { AR-3.5:1 } \\
\quad d: 80\end{array}$ & Size-dependent LSPR & 650 & $1.34-1.7$ & {$[82]$} \\
\hline Nanocrescents & $800-2,800$ & $\begin{array}{l}d: 410 \\
\theta: 10^{\circ} \\
\text { AR-4 }\end{array}$ & $\begin{array}{c}\text { Plasmon-induced } \\
\text { electromagnetic } \\
\text { near-field } \\
\end{array}$ & 879 & $\begin{array}{l}\text { Streptavidin } \\
\text { binding }\end{array}$ & [83] \\
\hline Gold nanorings & $900-1,500$ & $\begin{array}{c}d: 150 \\
t_{\text {Wall }}: 20\end{array}$ & $\begin{array}{c}\text { Shape dependence on } \\
\text { dielectric substrate }\end{array}$ & 880 & $1.33-1.42$ & {$[84]$} \\
\hline $\begin{array}{l}\text { Nanocross and } \\
\text { nanobar }\end{array}$ & $1200-1,900$ & $\begin{array}{l}L: 380 \\
W: 76 \\
\theta: 60^{\circ}\end{array}$ & $\begin{array}{l}\text { Subradiant Fano } \\
\text { resonances }\end{array}$ & $710-1,000$ & $1.333-1.38$ & {$[85]$} \\
\hline Nanohole & $450-950$ & $\begin{array}{c}d: 200 \\
p: 500 \\
t: 200(\mathrm{Au})\end{array}$ & EOT & 481 & $1.33-1.36$ & {$[86]$} \\
\hline Double-hole array & $500-680$ & $\begin{array}{c}d: 200 \\
\text { C-C spacing: } 190 \\
p: 500-600 \\
t: 100\end{array}$ & EOT-LSPR & 600 & $1-1.4$ & {$[76]$} \\
\hline $\begin{array}{l}\text { Silica sphere stripped } \\
\text { nanowell }\end{array}$ & $600-1000$ & $\begin{array}{c}d: 202-294 \\
h: 297-340 \\
p: 280-370 \\
t: 60(\mathrm{Au})\end{array}$ & LSPR & $1200-1600$ & $1-1.6$ & {$[45]$} \\
\hline $\begin{array}{l}\text { Nano-Lycurgus cup } \\
\text { array }\end{array}$ & $300-1100$ & $\begin{array}{c}d: 170 \\
h: 250-1000 \\
p: 350 \pm 30 \\
t: 60-120(\mathrm{Ag})\end{array}$ & $\begin{array}{c}\text { Mie } \\
\text { scattering-LSPR-EOT }\end{array}$ & 46000 & $1-1.56$ & {$[46]$} \\
\hline
\end{tabular}

$d$ : diameter; $h$ : height; $p$ : period; $t$ : thickness; $W$ : width; $L$ : length; $a$ : length of the side of cube; AR: aspect ratio; C-C: center-to-center; EOT: extraordinary optical transmission; LSPR: localized surface Plasmon resonance.

SPR sensor are a convolution of both surface and solutionbased refractive index changes. In general, LSPR-based sensors are at least an order of magnitude less sensitive than SPR sensors [49]. In addition, the smaller propagation lengths (or lifetimes) in typical LSPR sensors lead to broad resonance line width. This limits the figure of merit (FOM) achievable by LSPR sensors. However, in the nano-LCA sensor design the mirror-like structures such as Bragg reflectors were used to produce Fano resonances from the interference of evanescent (dark) and propagating (bright) modes (Figure 9) [46]. This allowed the sensor to have higher FOM in spite of using LSPR. True colorimetric sensing on subwavelength hole structures (also known as EOT devices) is challenging because of the appearance of multiple transmission peaks due to multiple interferences and diffraction of light generally known as the surface plasmon polariton-Bloch wave (SPPBW) and Wood's anomaly. The so-called quasi 3D plasmonic crystals [50] utilize the change in the transmission intensities (none or minimal shift in the resonance peak wavelength) for analyte detection and quantification. However, the resonance of such devices has only been demonstrated in near infrared and far infrared wavelengths [50] making it difficult to use conventional bright field microscopes and color photography for colorimetric sensing. On the other hand, the reported nano-LCA sensor demonstrates an improvement in the biosensing platform by combining LSPR and SPR. In this design LSPR scattering modes of the metal nanoparticles on the nanocup side walls are selectively transmitted by the nanohole array to achieve higher sensitivity.

The sensitivity $(S)$ of the SPR sensor is defined as the ratio between the resonance wavelength shift to the variation of the refractive index of the surrounding medium, $S=$ $\Delta \lambda(\mathrm{nm}) / \Delta n(\mathrm{RIU})$, and the FOM of the sensor is defined as the ratio between sensitivity and the line width of the resonance $(\delta \lambda)$ :

$$
\mathrm{FOM}=\frac{S\left(\mathrm{~nm} \cdot \mathrm{RIU}^{-1}\right)}{\delta \lambda(\mathrm{nm})} .
$$

Research studies are primarily pursued to increase the sensitivity (increasing the wavelength shift) and improve FOM (decreasing the line width) of the SPR sensor in addition to developing various biosensing applications. Table 1 presents a comparison of sensitivity of different plasmonic 
sensors. As shown in the table, one of the ways to increase sensitivity is by increasing the aspect ratio as the aspect ratio is directly proportional to the dipolar polarizability. For example, by increasing the aspect ratio from sphere to disk and to nanorods, the sensitivity generally improves. Similarly, the sensitivity can be improved by introducing multipolar resonances, for example, using nanocrescents [51, 52], instead of dipolar resonances (e.g., nanospheres). Peter Nordlander recognized that plasmon hybridization can be utilized, such as using nanorings, to increase the sensitivity of the SPR sensor [53]. Fano resonance, where weak coupling and interference occur between dark and bright plasmon modes, has emerged as another method to improve the sensitivity. In 1998, Ebbesen et al. showed that periodic nanohole arrays in thick metal film can be utilized to observe extraordinary optical transmission (EOT) [54]. Since then, the EOT structure has been extensively used for surface-based biosensing as opposed to colloidal-based sensing. In order to improve the sensitivity of EOT structures, LSPR, colloidal particles and mirror like structures have been incorporated [46].

Even though the naked eye sensing is implemented with plasmon-based colorimetry, it is for qualitative detection only. To make a biosensor which can do point-of-care detection and accurately perform quantitative analysis, especially for lab-on-chip applications, peripheral facilities and packaging are indispensable. The lab-on-a-chip-based sensor has the advantages of miniaturization, portability, automation, low cost, and negligible consumption of reagents. In recent decades, the development of microfluidic-based technology has greatly advanced the progress of micro total analysis systems [26]. The simultaneous rise of plasmonics and microfluidics has enabled the researchers in the fields enthusiastically to explore for plasmonic subwavelength optics and rapid-prototyping of lab-on-a-chip devices, forging a new field named plasmofluidics [55]. Among the plasmofluidic devices, ordered arrays of nanoholes in metal films facilitate resonance-induced field enhancement with no need of additional optical instrumentation [56]. Using nanohole arrays as sensing elements has the advantages of high reproducibility, small footprint, multiplexing capabilities, and the possibility for collinear optical integration [57]. These advantages make nanoholes particularly suitable for planar integration into onchip microfluidic environments [55].

\section{Microfluidic Nanohole Array Sensors}

Nanohole array sensors have been integrated with microfluidics to realize simple, portable, label-free optical sensors that have a small sample volume requirement, improved mass transport, and simplified optical illumination and detection systems. Microfluidics have been integrated with other types of label-free optical sensors such as surface plasmon resonance [58], photonic crystal [59], and ring resonator [60]. Affinity capture assays performed on a photonic-crystal sensor demonstrated that the microfluidic format has the fastest analyte detection time, the lowest limit of analyte detection, and the largest binding of analyte compared to the same assays performed in a microplate format and a spot format [61]. Moreover, microfluidic format has the advantages of enabling kinetic measurements (e.g., association and disassociation of analyte) and low analyte consumption. In particular to nanohole array sensors, microfluidics also allow for flow-through design, as opposed to flow-over, when the nanoholes are open through holes; in this case, the nanohole array acts not only as a sensing element but also as an analyte concentrator by confining the flow to the sensing area [62]. The integration of microfluidics to plasmonic-based sensors and, more specifically, nanohole-array-based sensors has been extensively reviewed elsewhere $[26,55]$.

\subsection{Fabrication, Implementation, and Characterization of Nanohole Array Sensors}

4.1.1. Fabrication and Integration of Microfluidics with Nanohole Array Sensor. (Poly)dimethylsiloxane (PDMS) is a common elastomer used to fabricate microfluidic devices due to its many advantages, including solvent compatibility, optical transparency, and feature replication (Figures 10(a)-10(c)) [63, 64]. In fabrication, the precursor agents (base agent and curing agent) of PDMS are mixed and degassed, and the PDMS solution is poured over a master with microfluidic design, which can be fabricated by photolithography or stereolithography. The PDMS solution is then cured thermally, and after curing, the microfluidic design is replicated on the PDMS substrate. The PDMS can be used to replicate microscale features (e.g., channels and chambers) down to hundreds of nanometers in a lateral resolution reliably and the same master can be used to fabricate more PDMS devices repeatedly [65]. Most commonly, the PDMS devices are integrated with a $\mathrm{SiO}_{2}$ surface, in which both surfaces are treated with $\mathrm{O}_{2}$ plasma to form hydroxyl groups and to form chemical adhesion between the two surfaces [66]. However, nanohole array sensors are typically coated with coinage metals, for example, gold ( $\mathrm{Au}$ ) or silver (Ag), and these materials are not compatible with $\mathrm{O}_{2}$ plasma treatment to integrate with the PDMS microfluidic device.

Other bonding techniques are available to integrate the PDMS microfluidic device with the nanohole array sensors (Figures 10(d)-10(f)) [67]. The desirable properties of the bonding method and materials are solvent compatibility, topographical compatibility, mechanical robustness, and optical transparency. A thin layer of silica has been deposited on top of the coinage metal for $\mathrm{O}_{2}$-plasma-treatment adhesion and biological interfacing [68]. Optical adhesives, curable by photo- or thermal activation, can act as an adhesive layer between the PDMS device and the surface of the nanohole array sensor [67]. For nonpermanent adhesion, the PDMS device has been mechanically compressed via sandwiching between two rigid plates and clamping [69]. Likewise, the nanohole array can be directly integrated into a flow cell, which can be fabricated with other materials beside PDMS [70]. Due to the flexibility in the channel designs and the ease of fabrication methods, the microfluidic device can be tailored to the applications ranging from simple 


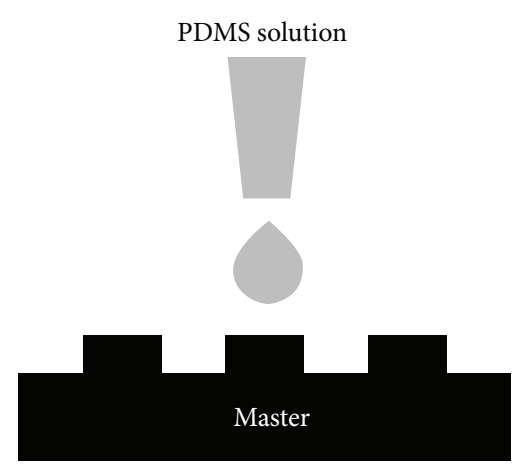

(a)

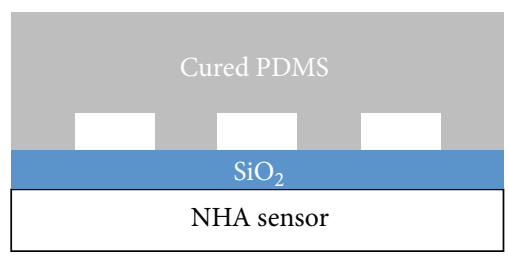

(d)

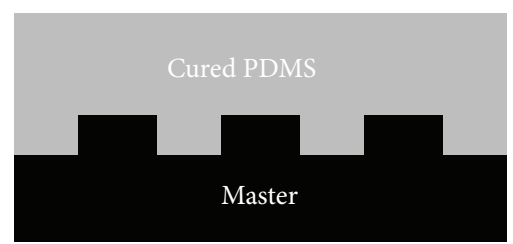

(b)

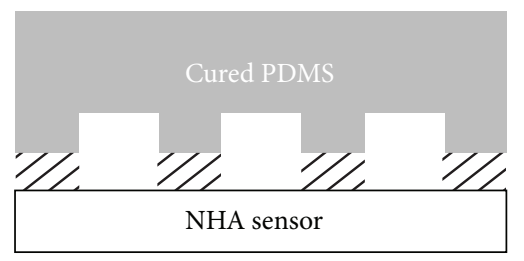

(e)

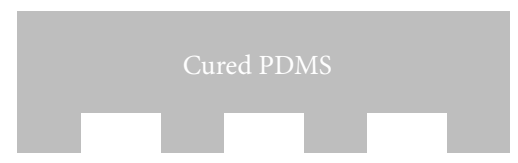

(c)

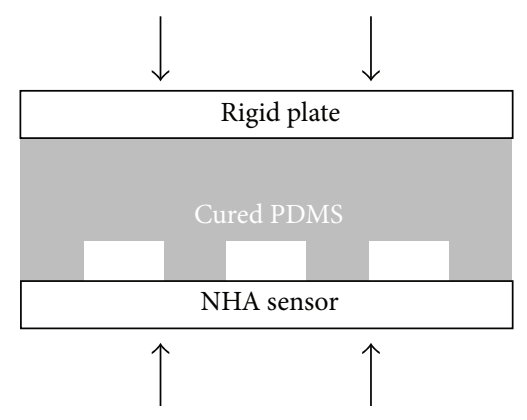

(f)

FIGURE 10: Schematic overview of fabrication and integration of PDMS-based microfluidic device with nanohole array (NHA) sensor. (a) (Poly)dimethylsiloxane (PDMS) solution consisted of the base agent and the curing agent and is poured over a master mold. ((b)-(c)) The solution is cured thermally and, when cured, the PDMS device is excised and removed from the master mold. The PDMS-based microfluidic device can be integrated with the nanohole array sensor via (d) deposition of thin layer of $\mathrm{SiO}_{2}$, (e) stamp of thin layer of adhesive (indicated by diagonal lines), and (f) compression between rigid plates. Note the deposited layers are not drawn to scale.

fluid transport to fluid mixing using serpentine channels (Figure 11).

4.1.2. Optical Illumination and Detection of Nanohole Array Sensors. An optical illumination source is required to observe the extraordinary transmission and to detect the changes in the intensity of the transmitted or reflected spectrum of the light as it transmits or reflects from the nanohole array. The optical illumination source is typically a broadband source (e.g., tungsten-halogen lamp, xenon arc lamp) with light production in the visible wavelength range $(\lambda \sim 450-750 \mathrm{~nm})$ and in the near-infrared range $(\lambda \sim 750-1000 \mathrm{~nm})$. A broad range of wavelength is required to detect the change in the wavelength at which there is maximum intensity. Alternatively, a monochromatic illumination source (e.g., laser, light emitting diode) can be utilized to illuminate the nanohole array but only the variations in the intensity of transmitted/reflected light can be detected. With monochromatic illumination sources, the choice of wavelength is limited due to the discretized nature of the light that can be produced (e.g., laser lines are limited wavelengths such as $532 \mathrm{~nm}$ and $633 \mathrm{~nm}$ ). An important property for an illumination source for nanohole array is high spectral irradiance (given in units of $\mathrm{W} / \mathrm{m}^{2} \mathrm{~nm}$ ) in the relevant range of wavelengths or high irradiance $\left(\mathrm{W} / \mathrm{m}^{2}\right)$ for monochromatic sources. Even with extraordinary transmission, nanohole array sensors typically transmit less than ten percent of the illuminated light; in addition, the area of the sensor may be limited to hundreds of micrometer squared, which requires high magnification objectives with a small field of view that reduces the amount of the transmitted light to be collected.

Accordingly, several optical detection systems have been used in conjunction with the aforementioned illumination sources. For spectral measurement, a grating is required in the detection system to disperse the broadband transmitted light and a charge-coupled detector is used to quantify the dispersed light according to its wavelength, for example, in a spectrophotometer (Figure 12(a)). Photomultiplier tubes may be used for quantifying the transmitted light from monochromatic illumination sources. Additional optical components such as high pass, low pass, band pass filters, and dichromatic mirrors may be employed to further distinguish the spectral or intensity changes in the transmitted light through the nanohole array sensor. For imaging, chargecoupled detectors overlaid with a color filter array (e.g., Bayer filter that has $50 \%$ green, $25 \%$ blue, and $25 \%$ red components) can be used to visualize the nanohole array sensor with some spectral distinction (Figure 12(b)). In our study, both forms of instruments schematically shown in Figure 12 were utilized.

4.1.3. Analysis of Detection Using Nanohole Array Sensors. In the case of broadband illumination, the spectrum of transmitted light through a nanohole array is quantified using 

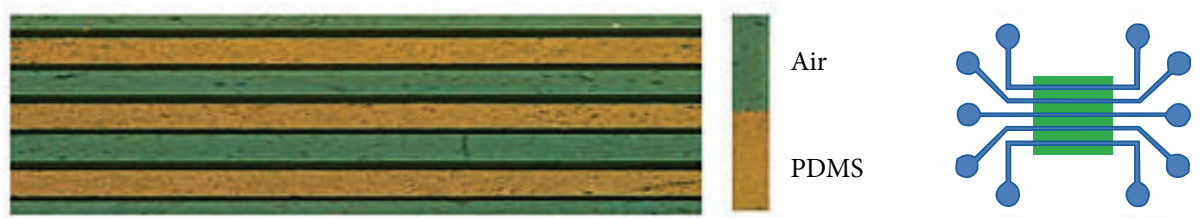

(a)

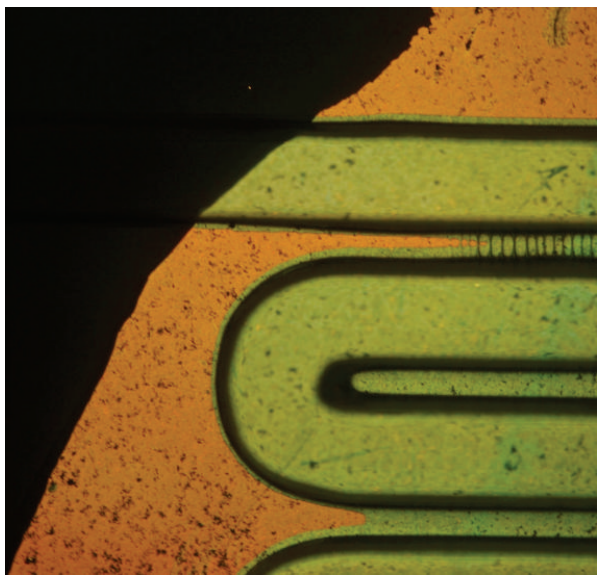

(b)

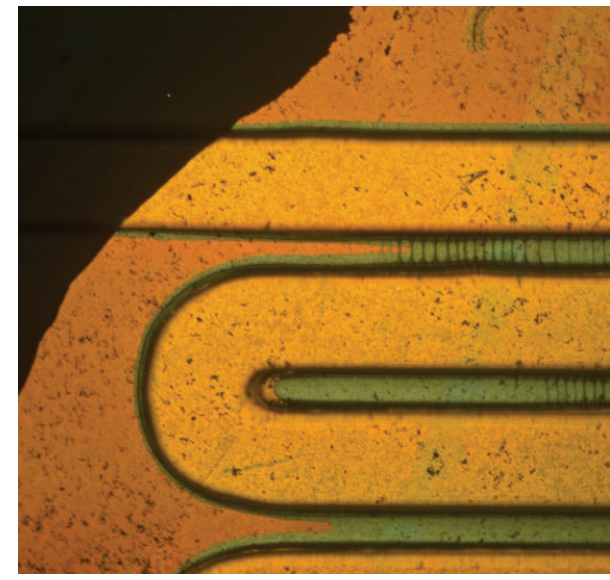

(c)

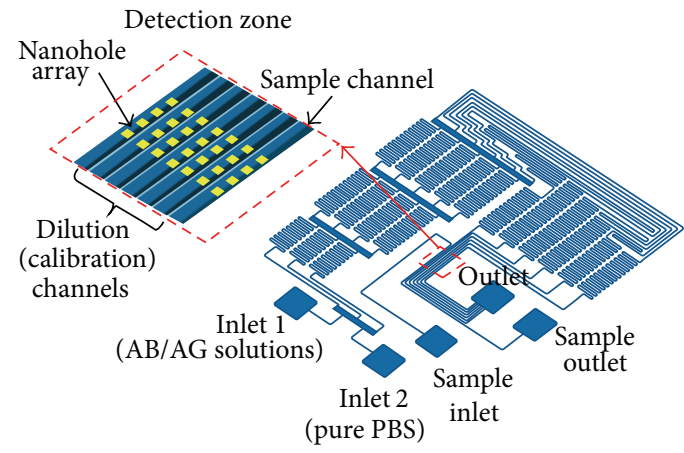

(d)

Figure 11: Overview of different microfluidic designs integrated on nanohole array sensor. (a) Parallel microfluidic channels with a width of $500 \mu \mathrm{m}$. (b) Serpentine microfluidic channels on a nanohole sensor array empty and (c) the same channel filled with water. (Channel width: $500 \mu \mathrm{m}$ ). (d) Microfluidic concentration gradient generator using serpentine channels integrated with nanohole array sensors. Modified from [77].

a spectrophotometer. In the measured spectrum, there may be single or multiple peak wavelengths, which is the wavelength at which the transmitted intensity is global or local maximum (Figure 13). The peak wavelength can be identified by simply searching the intensity maxima and the peak wavelength shift $(\Delta \lambda)$ can be quantified by determining the change in wavelength when the nanohole array is exposed to different refractive indices (Figure 13(a)). This peak wavelength shift can be correlated with the change in the refractive index $(\Delta n)$ on or adjacent to the surface of nanohole array sensor. The peak wavelength shift sensitivity $(S)$ of the nanohole array sensor can be calculated as $S=\Delta \lambda / \Delta n$. Other metrics can be used to quantify the changes in the transmission spectrum (spectral or intensity). One method is to integrate the area under the transmitted spectrum under a range of wavelengths to obtain integrated response sensitivity $(R)$ [71]. For a given spectrum, $R$ can be calculated as $R(n)=\sum_{\lambda_{1}}^{\lambda_{2}} \mid(I(n, \lambda)-$ $\left.I\left(n_{0}, \lambda\right)\right) / I\left(n_{0}, \lambda\right) \mid \times 100 \%$, where $I(n, \lambda)$ is the measured intensity at wavelength $\lambda$ for a given refractive index $n\left(n_{0}\right.$ is the reference refractive index), and $\lambda_{1}$ and $\lambda_{2}$ are the wavelength range over which the spectrum is integrated (Figure 13(b)). This method of sensitivity measurement can have a higher signal-to-noise ratio compared to the peak wavelength shift measurement [71]. For intensity variation measurement interrogated by a monochromatic light source, a single value is obtained for a single point measurement and a time trace can be obtained by recording the intensity over a course of time period (Figure 13(c)). For colorimetric imaging measurement, the red, green, and blue (RGB) 


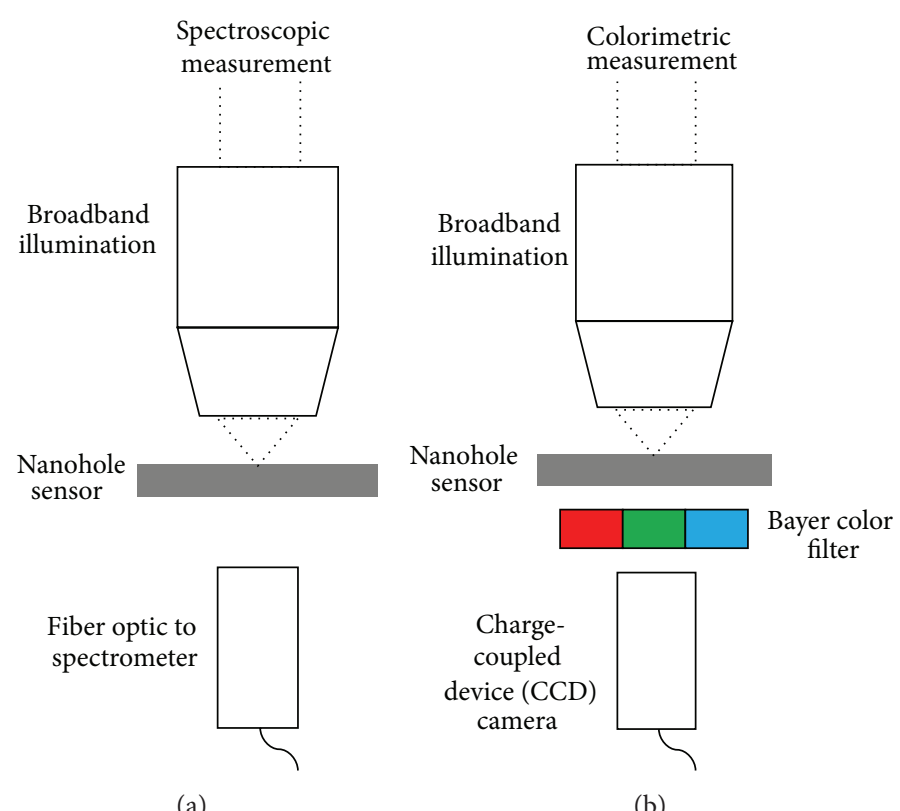

(a)

(b)

FIGURE 12: Typical optical setup for the measurement of transmission through nanohole sensor. (a) In spectroscopic measurement, in which the spectrum of the transmitted light is acquired, a broadband illumination is incident on the nanohole sensor and the transmitted light is collected using a fiber optic coupled to a spectrometer. (b) In colorimetric measurements, in which the intensity of the transmitted light through a color filter (e.g., Bayer color filter) is acquired, a broadband illumination is incident on the nanohole sensor, and the transmitted light is passed through a color filter and imaged using a charge-coupled device (CCD) camera.

channels from a color image can be obtained. Each channel represents a portion of the transmission spectrum; that is, the intensity in the red channel is from the transmitted light in a longer wavelength range and the intensity in the blue channel is from the transmitted light in a shorter wavelength range. Similar to intensity variation measurement, the change in the intensity from each channel can be correlated with the change in the transmission spectrum. Unlike intensity variation measurement, RGB contains three values for a single point measurement and it may have higher sensitivity than that of intensity variation measurement [72].

\subsection{Application of Microfluidic Nanohole Array with Biofluids}

4.2.1. Surface Functionalization of Nanohole Array Sensor. Like other label-free sensors, the nanohole array sensor requires a selective surface layer, which needs to be functionalized, for specificity and for capturing the analyte of interest and preventing nonspecific binding of other analytes (Figure 14(a)). The desired properties of surface functionalization are high surface density, high stability (i.e., temperature, solvent, flow rate), high analyte capture efficiency (binding affinity), and low cross-reactivity. Different strategies for surface functionalization have been reviewed, in the context of label-free sensors, with emphasis on the type of material surface that requires functionalization $[73,74]$. Since nanohole array sensors have surfaces coated with coinage metals (e.g., $\mathrm{Au}$ or $\mathrm{Ag}$ ), there is a limitation to the surface functionalization method that can be employed. The most common method of surface functionalization is to utilize a thiol group, which contains a sulfur atom, to react with the surface atoms containing $\mathrm{Au}$ or $\mathrm{Ag}$. The reaction requires a molecule with thiol-terminated group (e.g., alkanethiol) to be incubated via immersion, which will then self-assemble on the surface of the sensor. By coupling the desired ligand on the opposite end of the molecule from the thiol-terminated group, the surface functionality can be specified. For example, for the traditional avidin/streptavidin-biotin affinity assay, the surface of the sensor can be functionalized with biotinylated thiol, which contains a thiol group on one end and biotin on the other. The thiol group can react with the surface of the sensor forming a covalent bond and the biotin group is available to bind with the avidin/streptavidin molecule when introduced over the sensor. For more complicated surface functionalization, other reactive moieties can be functionalized to react with the ligand of interest. For example, cysteamine, which contains a thiol group and an amine group, can be functionalized on the surface of the sensor first. The amine group can further react with an activated carboxyl group (activated with EDC/NHS chemistry) available on the surface of a protein of interest.

\subsubsection{Affinity Capture Assays Using Nanohole Array Sensor}

DNA Hybridization. Nanohole array sensors have been used to detect the hybridization of complementary oligonucleotide strands with 18 base pairs [46]. The probe oligonucleotide strand is thiol-terminated at the $5^{\prime}$ end and is immobilized to the coinage-metal sensor surface. The target oligonucleotide strand contains an exact match complementary sequence to that of the probe strand and is labeled with a fluorophore (HEX) to confirm the hybridization to the probe strand. 


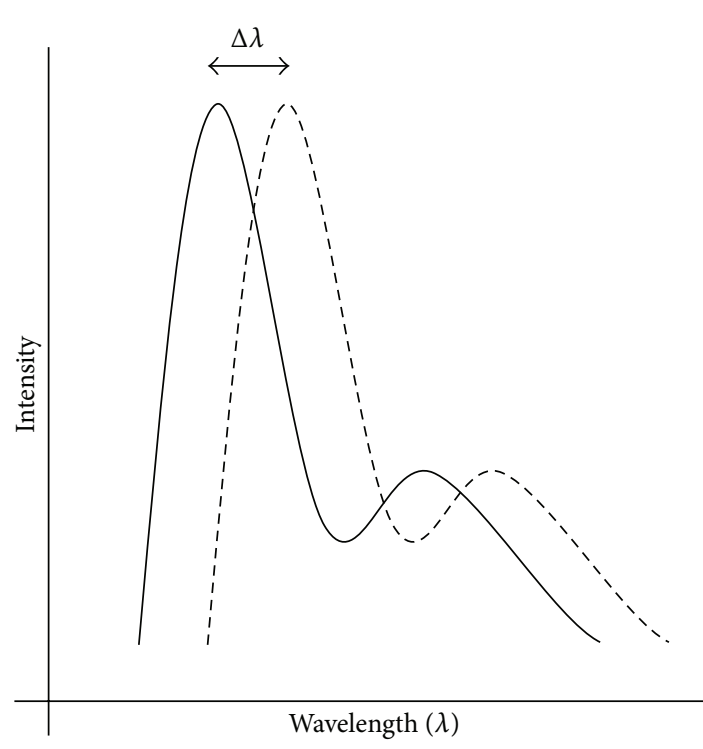

(a)

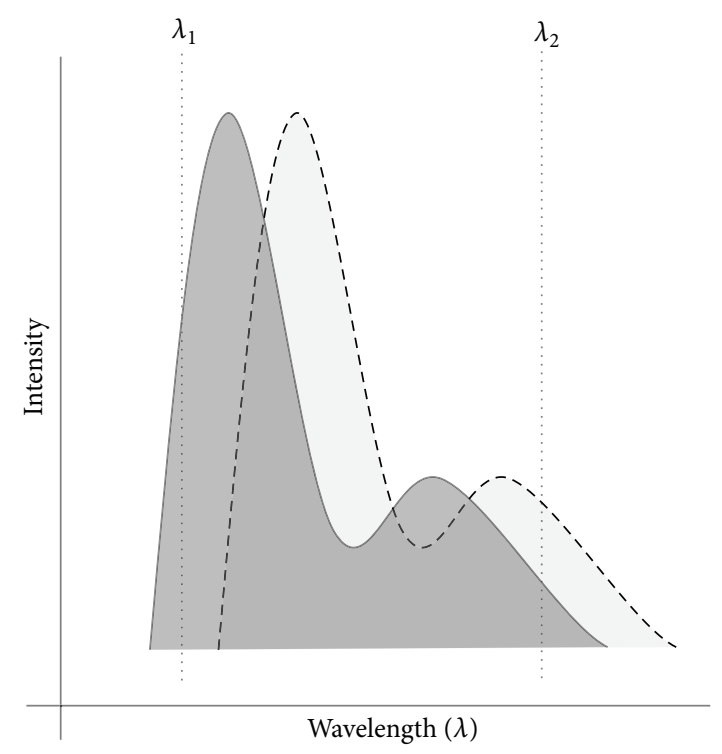

(b)

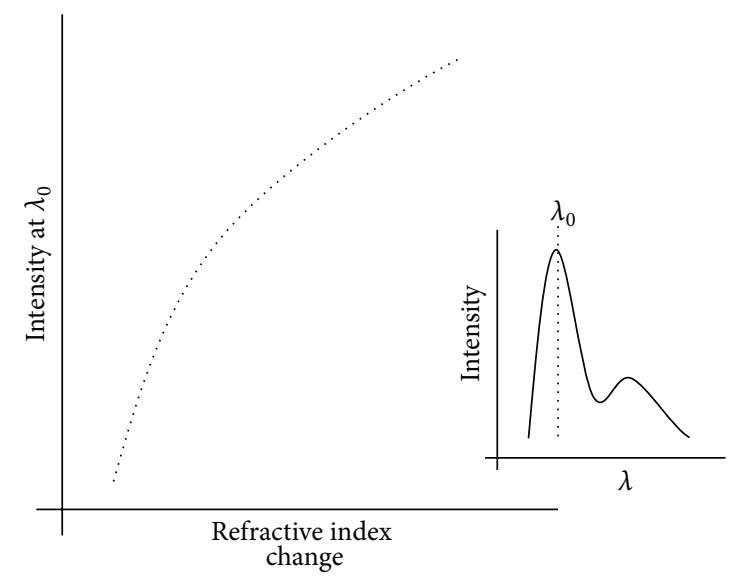

(c)

FIGURE 13: Schematic representative of the optical response of nanohole array sensor to refractive index change. (a) The change in the wavelength of maximum transmission intensity, also known as peak wavelength shift, can be quantified when comparing the transmission spectrum of nanohole array sensor exposed water (refractive index $=1.33$ ) (solid) and water with glucose added (refractive index $=1.34$ ) (dashed). (b) Integrated sensor response can be obtained from integrating the area under the transmission spectrum over a defined range of wavelengths (e.g., $\lambda_{1}$ to $\lambda_{2}$ ). (c) Intensity variations at a single wavelength from the transmission spectrum (inset) can also be monitored in response to changes in the refractive index on the nanohole array sensor.

The hybridization of $10 \mu \mathrm{M}$ of the target strand to the immobilized probe strand $(100 \mu \mathrm{M})$ induced approximately $32 \mathrm{~nm}$ in the peak wavelength shift and could be observed colorimetrically with an increase in the intensity of the red channel down to $0.1 \mathrm{nM}$ of the target strand, confirmed by fluorescence imaging (Figure 14(c)).

Nanohole array sensors can also be used to detect color change in response to difference in DNA concentration in solution form as shown in Figure 15. For 100 pM of DNA solution on the sensor, the transmission image looks greenish orange, whereas the $100 \mu \mathrm{M}$ DNA solution looks red on the transmission image. The color spectra obtained from the image clearly show the red shift trend of the resonance with an increasing concentration of DNA solution.
Antigen-Antibody Binding. Antigen-antibody binding assay is commonly used to demonstrate the performance of the sensor by determining the limit of detection of the sensor and the association constant, $K_{a}$, of the antigen-antibody binding. This assay has high relevance in the pharmaceutical industry where large libraries of molecules need to be screened against potential drug targets for binding affinity. A common model binding assay is the biotin-avidin model, where the biotin molecules (molecular weight: 244 Daltons) can bind with the four available binding sites of one avidin molecule (molecular weight: 60 kiloDaltons) [75]. The $K_{a}$ of the biotin-avidin interaction is approximately $10^{15}$ molar $^{-1}$. The detection of the interaction between biotin avidin has been demonstrated using nanohole array sensors $[35,69,72]$. In particular, 

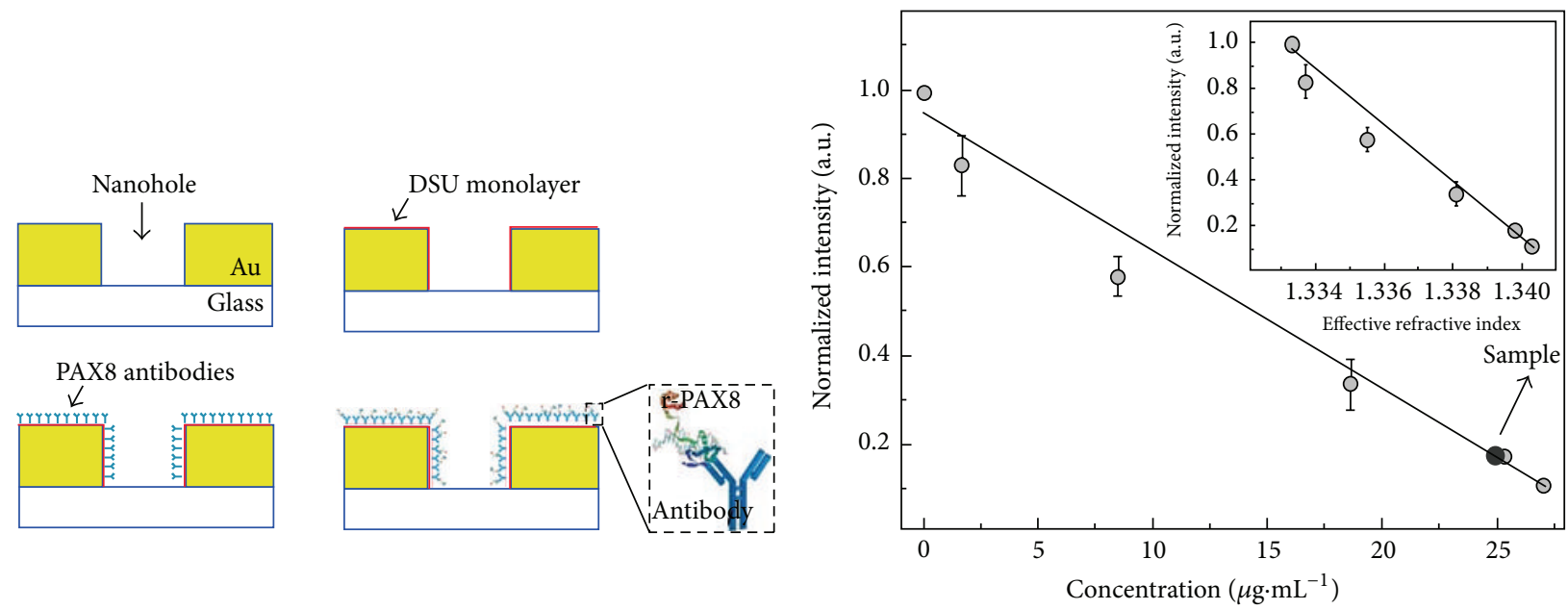

(a)

(b)

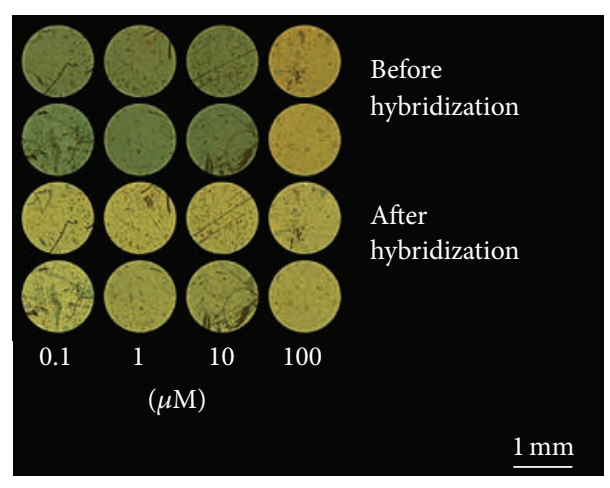

(c)

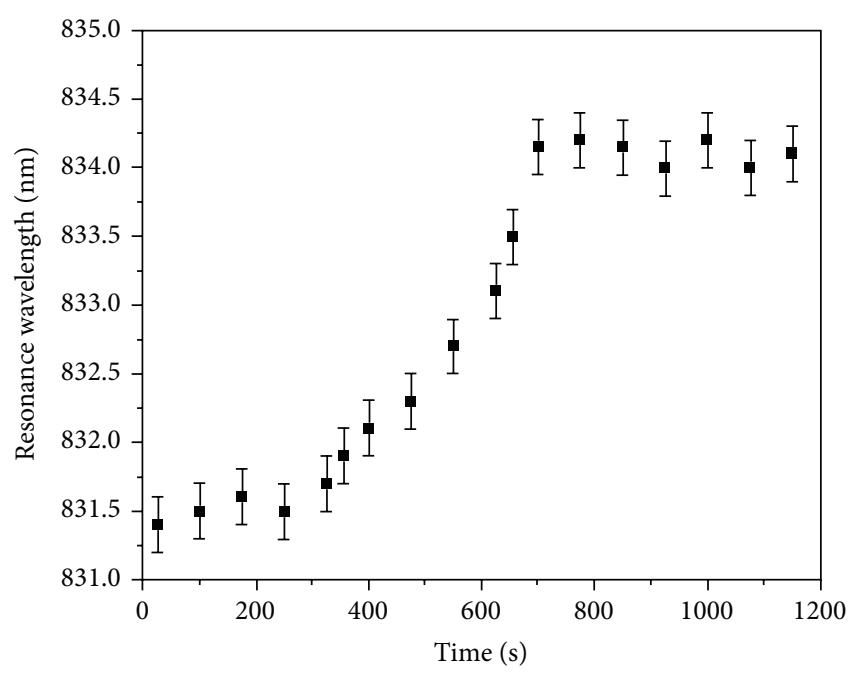

(d)

FIGURE 14: Surface functionalization and detection of biomolecules using nanohole array sensor. (a) Typical surface modification of nanohole array sensor for specific recognition of biomolecule. The surface is modified with a monolayer of linker (DSU) and antibodies (PAX8). (b) Intensity variation due to the presence of increasing concentrations of antibodies on the surface of the nanohole array sensor. Modified from [77]. (c) Colorimetric variations due to difference concentrations of target and probe DNA strands immobilized from nanohole array sensor. Modified from [46]. (d) Peak wavelength shift due to the nonspecific attachment of bovine serum albumin on a nanohole array sensor. Modified from [93].

the binding of streptavidin (1 milligram dissolved in 4 milliliters of phosphate buffered solution) to immobilized cysteamine-linked biotin was observed to induce a peak wavelength shift of 3.5-4 nanometer [69]. Another model molecule for protein is bovine serum albumin (molecular weight: 66.5 kiloDaltons), which can be immobilized on the sensor by simple protein adsorption or covalently linked by carboxylated thiol linker (e.g., 6-mercaptohexanoic acid). Simple protein adsorption of bovine serum albumin on a nanohole array sensor had a peak wavelength response of 3 nanometers [46], while on a different nanohole array sensor, anti-BSA antibody binding to BSA induced a peak wavelength shift of 42 nanometers (Figure 14(d)) $[46,76]$. A biomarker of ovarian cancer (r-PAX8) was detected using immobilized antibodies (linked with dithiobissuccinimidyl undecanoate to the surface) on a nanohole array sensor (Figure 14(b)) [77]. The sensor had a limit of detection of 5 nanomolars within a range of 0.25 to $9.0 \mu \mathrm{g} \mathrm{mL}^{-1}$ using an intensity variation detection with a monochromatic illumination source at 633 nanometers. The binding of a monolayer of mouse IgG immunoglobulins to protein $\mathrm{A} / \mathrm{G}$ to a nanohole array caused a peak wavelength shift of 22 nanometers and when incorporated with a notch filter, the change in the peak wavelength shift could be visualized due to the corresponding change in the intensity [78].

4.2.3. Viral Detection. In a flow-through design, synthetic particles that are on the order of viruses (70 nanometer 


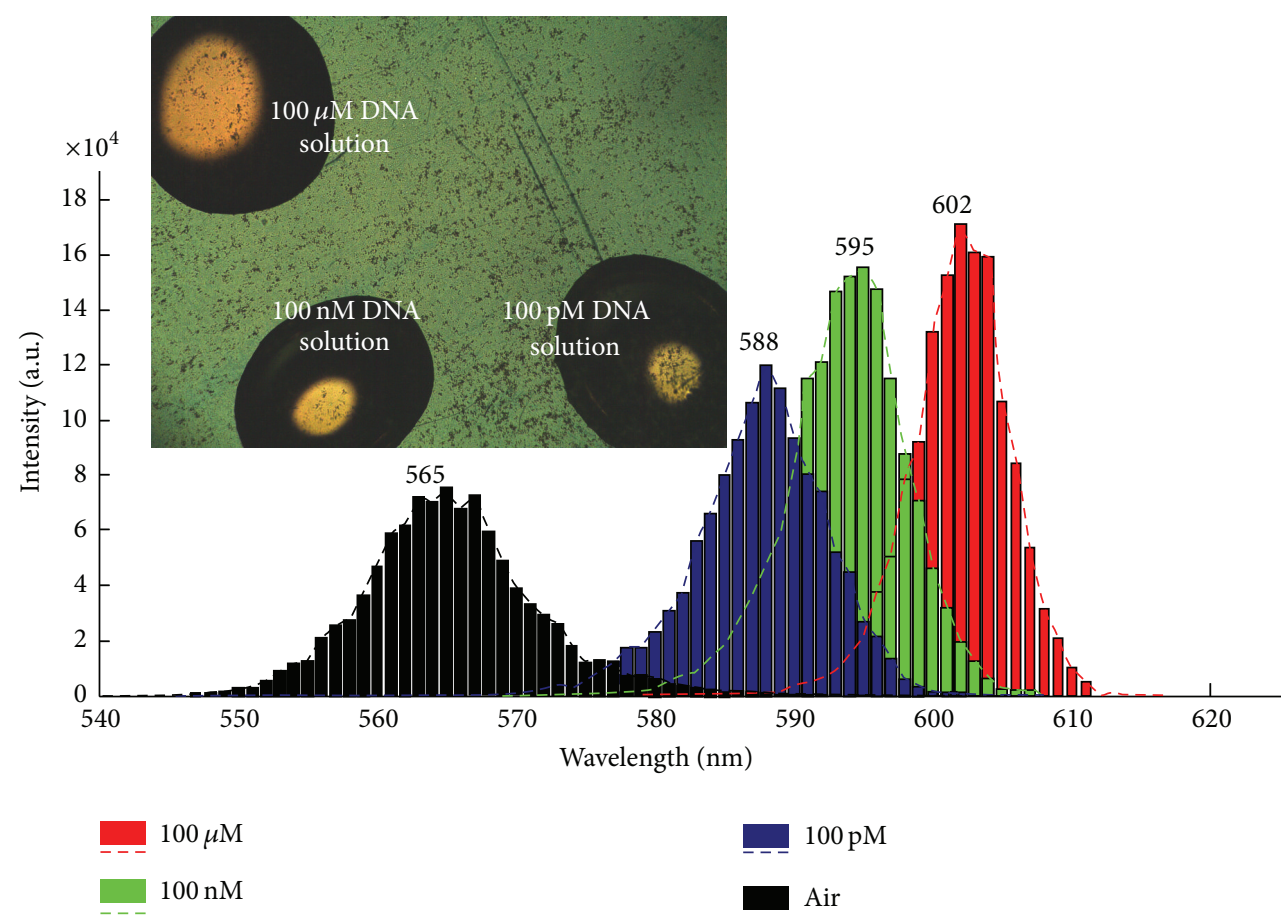

FIGURE 15: Solution-based colorimetric sensing using nano-LCA sensor. The inset image shows drop of DNA solution with concentration $100 \mathrm{pM}, 100 \mathrm{nM}$, and $100 \mu \mathrm{M}$ on the sensor surface. The transmission color of the sensor changes from yellow to red when DNA concentration changes from $100 \mathrm{pM}$ to $100 \mu \mathrm{M}$, respectively. The color spectra obtained from the image are also shown.

diameter polystyrene beads coated with streptavidin) have been detected using biotin-immobilized nanohole array sensor [70]. The surface of the sensor was functionalized with biotinylated thiol groups and the detection is due to the binding of the streptavidin-coated beads to the biotinylated surface of the sensor. The flow-through design provided a faster response time that is a peak wavelength shift of 15 nanometers observed in 40 minutes whereas the flowover design required 200 minutes to observe the same shift. Analyte concentration as low as $10^{3}$ particles $\mathrm{mL}^{-1}$ induced a measurable peak wavelength shift on the nanohole array sensor. Viral loads that consisted of vesicular stomatitis virus (VSV), Ebola virus, and vaccinia virus were detected on nanohole array sensor (periodicity: $600 \mathrm{~nm}$, hole radius: $220 \mathrm{~nm}$ ) with a flow-through design (Figures 16(a)-16(h)) [79]. For surface functionalization, protein $A / G$ was first immobilized directly on the surface of the sensor followed by antibodies specific to the glycoprotein presented on the surface of a particular virus. The binding of the protein $\mathrm{A} / \mathrm{G}$ and the antibodies caused a measurable shift in the peak wavelength ( $\sim \mathrm{nm}$ and $\sim 14 \mathrm{~nm}$, resp.). Upon binding of the viruses to the antibodies, a shift of approximately 14$21 \mathrm{~nm}$ peak wavelength shift was observed for the Ebola virus detection [79]. Even with the background interference from cell growth medium with fetal calf serum, viral load as low as $10^{6}$ plaque-forming unit (PFU) could be resolved compared to that of the reference sensor.
Future Outlook. Label-free plasmonic sensors, based on surface plasmon resonance (SPR) and localized surface plasmon resonance (LSPR), their microfabrication, instrumentations, integration to microfluidics, and applications for biofluids detection have been reviewed. The advancement of $3 \mathrm{D}$ nanohole fabrication techniques led to two orders of magnitude improvement in the FOM compared to colloidal nanoparticles [46]. The nanoimprint-based fabrication method enabled the formation of 3D nanohole array with footprint of four-inch wafers with a lower cost [46]. Nanohole array has been designed for both spectroscopic and color imaging-based on-chip biosensing applications. It has been shown that a flow-through microfluidic integrated nanohole sensor can improve the response time by 14 -fold compared to same sensor with flow-over sensing methods [26]. The nanohole array sensor, due to its advantages, will help realize portable, inexpensive, label-free, and plasmon-based sensors with high sensitivity and high usability point-of-care diagnostics. In particular, colorimetric nanoplasmonic sensor combined with microfluidics can be integrated with existing mobile electronics such as cell phones and tablets or with existing bedside equipment such as drug infusion and blood transfusion apparatus. Multiplexed molecular detections in biofluidic samples including blood plasma, urine, and saliva can be done with a simple colorimetric sensing on the nanoplasmonic sensor and yet at a much higher sensitivity and surface specificity in comparison with a chromophore-based colorimetric 


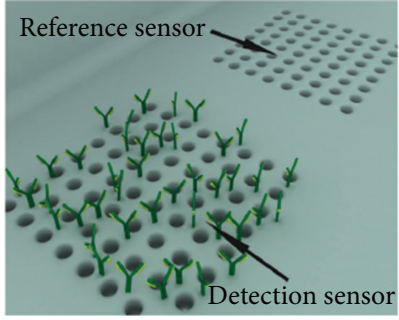

(a)

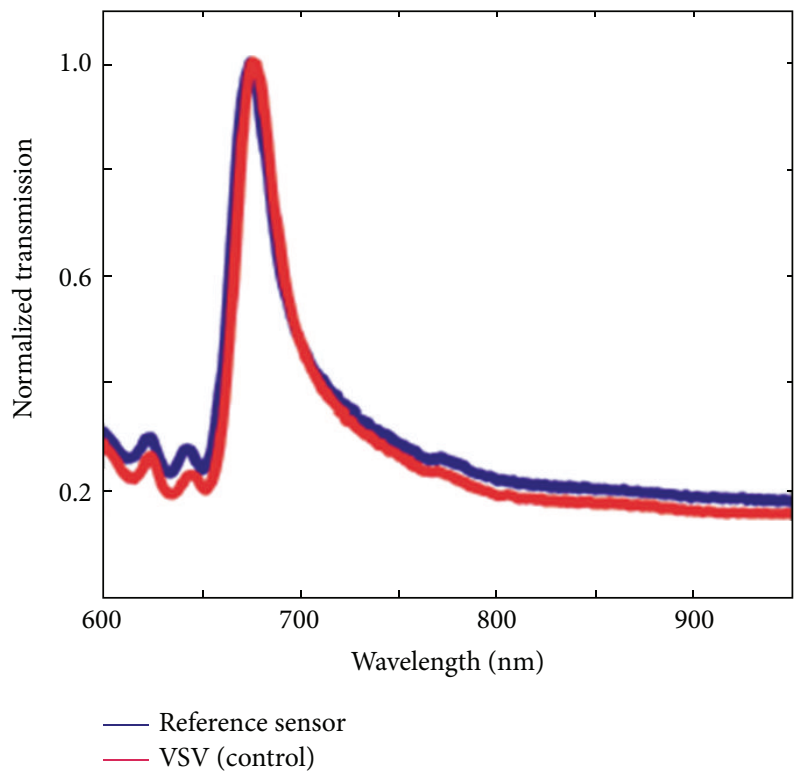

(c)

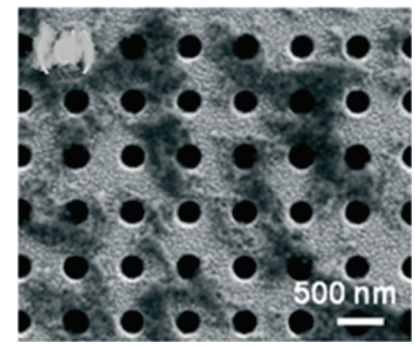

(e)

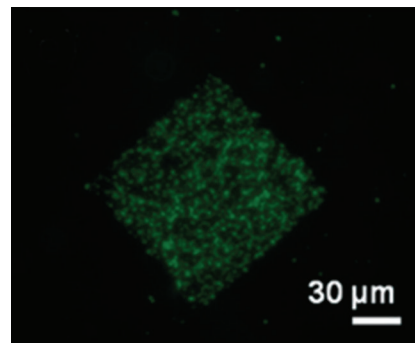

(f)

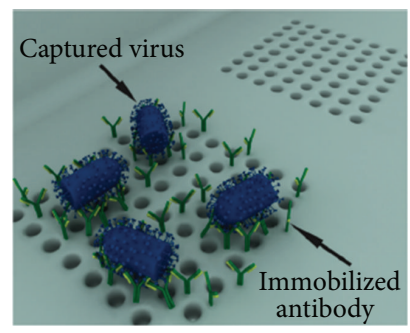

(b)

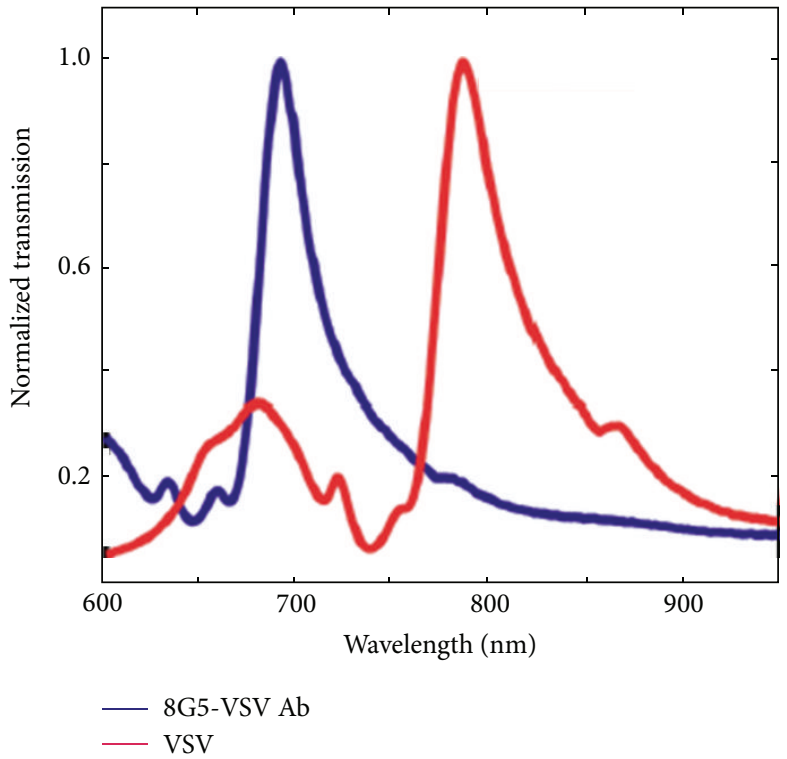

(d)

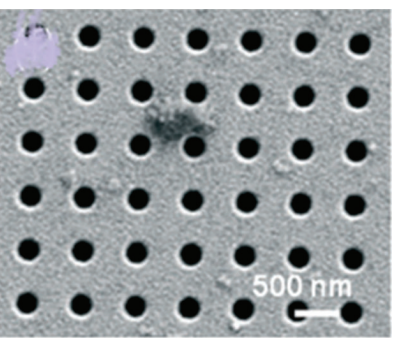

(g)

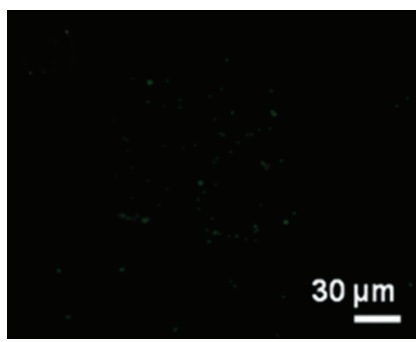

(h)

FIGURE 16: (a) Schematic showing nanohole plasmonic sensor functionalized (immobilized with capturing antibody targeting vesicular stomatitis virus (VSV)) and control sensors (unfunctionalized). (b) Schematic showing VSV attaches only to the antibody immobilized sensor. (c) Transmission spectrum of control sensor without the capture antibody. (d) Transmission spectrum of sensor with the capture antibody showing about $100 \mathrm{~nm}$ shift in the resonance peak after the capture of VSV. Adapted from [79] (e) SEM image of the sensor without the virus. (f) Optical image showing the transmitted color by the sensor without the virus. (g) SEM image of the sensor with the virus. (h) Optical image showing the transmitted color is vanished with the capture of virus. Adapted from [70].

assay. The nearly equipment-free nanoplasmonic sensing will completely transform the applications of SPR technology into new territories such as point-of-care diagnostics and telemedicine.

\section{Conflict of Interests}

The authors declare that there is no conflict of interests regarding the publication of this paper.

\section{Authors' Contribution}

Abid Ameen, Manas Ranjan Gartia, and Austin Hsiao contributed equally to this paper.

\section{References}

[1] R. M. Lequin, "Enzyme immunoassay (EIA)/enzyme-linked immunosorbent assay (ELISA)," Clinical Chemistry, vol. 51, no. 12, pp. 2415-2418, 2005. 
[2] H. K. Hunt and A. M. Armani, "Label-free biological and chemical sensors," Nanoscale, vol. 2, no. 9, pp. 1544-1559, 2010.

[3] "Surface plasmon resurrection," Nature Photonics, vol. 6, no. 11, p. 707, 2012.

[4] J. N. Anker, W. P. Hall, O. Lyandres, N. C. Shah, J. Zhao, and R. P. van Duyne, "Biosensing with plasmonic nanosensors," Nature Materials, vol. 7, no. 6, pp. 442-453, 2008.

[5] S. Singh, S. Gupta, A. K. Gupta, M. Singh, and A. Kumar, "Surface plasmon resonance sensogram based characterization of antibodies raised against intact teliospores and purified diagnostic antigen for development of nano-immunosensor for fungal spore antigen of karnal bunt (Tilletia indica) of wheat," Proceedings of the National Academy of Sciences, India Section B: Biological Sciences, vol. 83, pp. 551-560, 2013.

[6] B. Liedberg, C. Nylander, and I. Lunström, "Surface plasmon resonance for gas detection and biosensing," Sensors and Actuators, vol. 4, pp. 299-304, 1983.

[7] A. Aubé, J. Breault-Turcot, P. Chaurand, J. N. Pelletier, and J.-F. Masson, "Non-specific adsorption of crude cell lysate on surface plasmon resonance sensors," Langmuir, vol. 29, no. 32, pp. 1014110148, 2013.

[8] W. Qi, J. Zhao, W. Zhang et al., "Visual and surface plasmon resonance sensor for zirconium based on zirconium-induced aggregation of adenosine triphosphate-stabilized gold nanoparticles," Analytica Chimica Acta, vol. 787, pp. 126-131, 2013.

[9] Y. Yanase, T. Hiragun, T. Yanase, T. Kawaguchi, K. Ishii, and M. Hide, "Application of SPR imaging sensor for detection of individual living cell reactions and clinical diagnosis of type I allergy," Allergology International, vol. 62, no. 2, pp. 163-169, 2013.

[10] J.-Y. Byun, Y.-B. Shin, T. Li et al., "The use of an engineered single chain variable fragment in a localized surface plasmon resonance method for analysis of the C-reactive protein," Chemical Communications, vol. 49, no. 82, pp. 9497-9499, 2013.

[11] Y. Xia, J. Ye, K. Tan, J. Wang, and G. Yang, "Colorimetric visualization of glucose at the submicromole level in serum by a homogenous silver nanoprism-glucose oxidase system," Analytical Chemistry, vol. 85, no. 13, pp. 6241-6247, 2013.

[12] S. Chen, M. Svedendahl, R. P. V. Duyne, and M. Käll, "Plasmonenhanced colorimetric ELISA with single molecule sensitivity," Nano Letters, vol. 11, no. 4, pp. 1826-1830, 2011.

[13] Z. Xu, Y. Chen, M. R. Gartia, J. Jiang, and G. L. Liu, "Surface plasmon enhanced broadband spectrophotometry on black silver substrates," Applied Physics Letters, vol. 98, no. 24, Article ID 241904, 2011.

[14] Z. Xu, M. R. Gartia, C. J. Choi et al., "Quick detection of contaminants leaching from polypropylene centrifuge tubes with surface-enhanced Raman spectroscopy and ultraviolet absorption spectroscopy," Journal of Raman Spectroscopy, vol. 42, no. 11, pp. 1939-1944, 2011.

[15] P. K. Roy, Y.-F. Huang, and S. Chattopadhyay, "Detection of melamine on fractals of unmodified gold nanoparticles by surface-enhanced Raman scattering," Journal of Biomedical Optics, vol. 19, no. 1, p. 011002, 2014.

[16] A. F. Chrimes, K. Khoshmanesh, S.-Y. Tang et al., "In situ SERS probing of nano-silver coated individual yeast cells," Biosensors and Bioelectronics, vol. 49, pp. 536-541, 2013.

[17] P. D. Howes, S. Rana, and M. M. Stevens, "Plasmonic nanomaterials for biodiagnostics," Chemical Society Reviews, vol. 43, no. 11, pp. 3835-3853, 2014.
[18] K. M. Mayer and J. H. Hafner, "Localized surface plasmon resonance sensors," Chemical Reviews, vol. 111, no. 6, pp. 38283857, 2011.

[19] B. Sepúlveda, P. C. Angelomé, L. M. Lechuga, and L. M. LizMarzán, “LSPR-based nanobiosensors," Nano Today, vol. 4, no. 3, pp. 244-251, 2009.

[20] E. Petryayeva and U. J. Krull, "Localized surface plasmon resonance: nanostructures, bioassays and biosensing-a review," Analytica Chimica Acta, vol. 706, no. 1, pp. 8-24, 2011.

[21] T. Chung, S.-Y. Lee, E. Y. Song, H. Chun, and B. Lee, "Plasmonic nanostructures for nano-scale bio-sensing," Sensors, vol. 11, no. 11, pp. 10907-10929, 2011.

[22] M.-C. Estevez, M. A. Otte, B. Sepulveda, and L. M. Lechuga, "Trends and challenges of refractometric nanoplasmonic biosensors: a review," Analytica Chimica Acta, vol. 806, pp. 5573, 2014.

[23] K. A. Willets and R. P. van Duyne, "Localized surface plasmon resonance spectroscopy and sensing," Annual Review of Physical Chemistry, vol. 58, no. 1, pp. 267-297, 2007.

[24] J. Homola, "Surface plasmon resonance sensors for detection of chemical and biological species," Chemical Reviews, vol. 108, no. 2, pp. 462-493, 2008.

[25] G. Spoto and M. Minunni, "Surface plasmon resonance imaging: what next?" Journal of Physical Chemistry Letters, vol. 3, no. 18, pp. 2682-2691, 2012.

[26] C. Escobedo, "On-chip nanohole array based sensing: a review," Lab on a Chip-Miniaturisation for Chemistry and Biology, vol. 13, no. 13, pp. 2445-2463, 2013.

[27] O. Tokel, F. Inci, and U. Demirci, "Advances in plasmonic technologies for point of care applications," Chemical Reviews, vol. 114, no. 11, pp. 5728-5752, 2014.

[28] A. Krishnan, T. Thio, T. J. Kim et al., "Evanescently coupled resonance in surface plasmon enhanced transmission," Optics Communications, vol. 200, no. 1-6, pp. 1-7, 2001.

[29] H. F. Ghaemi, T. Thio, D. E. Grupp, T. W. Ebbesen, and H. J. Lezec, "Surface plasmons enhance optical transmission through subwavelength holes," Physical Review B, vol. 58, no. 11, pp. 6779-6782, 1998.

[30] C. Genet and T. W. Ebbesen, "Light in tiny holes," Nature, vol. 445, no. 7123, pp. 39-46, 2007.

[31] N. G. Khlebtsov and L. A. Dykman, "Optical properties and biomedical applications of plasmonic nanoparticles," Journal of Quantitative Spectroscopy and Radiative Transfer, vol. 111, no. 1, pp. 1-35, 2010.

[32] Y. Xia, X. Xia, Y. Wang, and S. Xie, "Shape-controlled synthesis of metal nanocrystals," MRS Bulletin, vol. 38, no. 4, pp. 335-344, 2013.

[33] J. Henzie, J. Lee, M. H. Lee, W. Hasan, and T. W. Odom, "Nanofabrication of plasmonic structures," Annual Review of Physical Chemistry, vol. 60, pp. 147-165, 2009.

[34] W. Chen and H. Ahmed, "Fabrication of 5-7 nm wide etched lines in silicon using $100 \mathrm{keV}$ electron-beam lithography and polymethylmethacrylate resist," Applied Physics Letters, vol. 62, no. 13, pp. 1499-1501, 1993.

[35] H. Im, S. H. Lee, N. J. Wittenberg et al., "Template-stripped smooth Ag nanohole arrays with silica shells for surface plasmon resonance biosensing," ACS Nano, vol. 5, no. 8, pp. 62446253, 2011.

[36] K. Kumar, H. Duan, R. S. Hegde, S. C. W. Koh, J. N. Wei, and J. K. W. Yang, "Printing colour at the optical diffraction limit," Nature Nanotechnology, vol. 7, no. 9, pp. 557-561, 2012. 
[37] N. C. Lindquist, P. Nagpal, K. M. McPeak, D. J. Norris, and S. $\mathrm{Oh}$, "Engineering metallic nanostructures for plasmonics and nanophotonics," Reports on Progress in Physics, vol. 75, no. 3, Article ID 036501, 2012.

[38] B. D. Gates, Q. Xu, J. C. Love, D. B. Wolfe, and G. M. Whitesides, "Unconventional nanofabrication," Annual Review of Materials Research, vol. 34, no. 1, pp. 339-372, 2004.

[39] B. D. Gates, Q. Xu, M. Stewart, D. Ryan, C. G. Willson, and G. M. Whitesides, "New approaches to nanofabrication: molding, printing, and other techniques," Chemical Reviews, vol. 105, no. 4, pp. 1171-1196, 2005.

[40] Y. Xia and G. M. Whitesides, "Soft lithography," Annual Review of Materials Science, vol. 28, no. 1, pp. 153-184, 1998.

[41] A. Boltasseva, "Plasmonic components fabrication via nanoimprint," Journal of Optics A: Pure and Applied Optics, vol. 11, no. 11, Article ID 114001, 2009.

[42] P. Nagpal, N. C. Lindquist, S.-H. Oh, and D. J. Norris, "Ultrasmooth patterned metals for plasmonics and metamaterials," Science, vol. 325, no. 5940, pp. 594-597, 2009.

[43] M. R. Gartia, Z. Xu, E. Behymer et al., "Rigorous surface enhanced Raman spectral characterization of large-area highuniformity silver-coated tapered silica nanopillar arrays," Nanotechnology, vol. 21, no. 39, Article ID 395701, 2010.

[44] A. Fernandez, H. T. Nguyen, J. A. Britten et al., "Use of interference lithography to pattern arrays of submicron resist structures for field emission flat panel displays," Journal of Vacuum Science and Technology B: Microelectronics and Nanometer Structures, vol. 15, no. 3, pp. 729-735, 1997.

[45] S. Y. Lee, S.-H. Kim, S. G. Jang, C.-J. Heo, J. W. Shim, and S.M. Yang, "High-fidelity optofluidic on-chip sensors using welldefined gold nanowell crystals," Analytical Chemistry, vol. 83, no. 23, pp. 9174-9180, 2011.

[46] M. R. Gartia, A. Hsiao, A. Pokhriyal et al., "Colorimetric plasmon resonance imaging using nano lycurgus cup arrays," Advanced Optical Materials, vol. 1, no. 1, pp. 68-76, 2013.

[47] L. S. Jung, C. T. Campbell, T. M. Chinowsky, M. N. Mar, and S. S. Yee, "Quantitative interpretation of the response of surface plasmon resonance sensors to adsorbed films," Langmuir, vol. 14, no. 19, pp. 5636-5648, 1998.

[48] A. J. Haes, S. Zou, G. C. Schatz, and R. P. van Duyne, "A nanoscale optical biosensor: the long range distance dependence of the localized surface plasmon resonance of noble metal nanoparticles," Journal of Physical Chemistry B, vol. 108, no. 1, pp. 109-116, 2004.

[49] A. Cattoni, P. Ghenuche, A.-M. Haghiri-Gosnet et al., " $\lambda^{3} / 1000$ plasmonic nanocavities for biosensing fabricated by soft UV nanoimprint lithography," Nano Letters, vol. 11, no. 9, pp. 35573563, 2011.

[50] M. E. Stewart, N. H. Mack, V. Malyarchuk et al., "Quantitative multispectral biosensing and $1 \mathrm{D}$ imaging using quasi-3D plasmonic crystals," Proceedings of the National Academy of Sciences of the United States of America, vol. 103, no. 46, pp. 17143-17148, 2006.

[51] B. M. Ross and L. P. Lee, "Plasmon tuning and local field enhancement maximization of the nanocrescent," Nanotechnology, vol. 19, no. 27, Article ID 275201, 2008.

[52] L. Yu, G. L. Liu, J. Kim, Y. X. Mejia, and L. P. Lee, "Nanophotonic crescent moon structures with sharp edge for ultrasensitive biomolecular detection by local electromagnetic field enhancement effect," Nano Letters, vol. 5, no. 1, pp. 119-124, 2005.
[53] E. Prodan, C. Radloff, N. J. Halas, and P. Nordlander, "A hybridization model for the plasmon response of complex nanostructures," Science, vol. 302, no. 5644, pp. 419-422, 2003.

[54] T. W. Ebbesen, H. J. Lezec, H. F. Ghaemi, T. Thio, and P. A. Wolff, "Extraordinary optical transmission through sub-wavelenght hole arrays," Nature, vol. 391, no. 6668, pp. 667-669, 1998.

[55] J. Kim, "Joining plasmonics with microfluidics: from convenience to inevitability," Lab on a Chip: Miniaturisation for Chemistry and Biology, vol. 12, no. 19, pp. 3611-3623, 2012.

[56] A. G. Brolo, R. Gordon, B. Leathem, and K. L. Kavanagh, "Surface plasmon sensor based on the enhanced light transmission through arrays of nanoholes in gold films," Langmuir, vol. 20, no. 12, pp. 4813-4815, 2004.

[57] D. Sinton, R. Gordon, and A. G. Brolo, "Nanohole arrays in metal films as optofluidic elements: progress and potential," Microfluidics and Nanofluidics, vol. 4, no. 1-2, pp. 107-116, 2008.

[58] V. Kodoyianni, "Label-free analysis of biomolecular interactions using SPR imaging," BioTechniques, vol. 50, no. 1, pp. 3240, 2011.

[59] C. J. Choi, I. D. Block, B. Bole, D. Dralle, and B. T. Cunningham, "Label-free photonic crystal biosensor integrated microfluidic chip for determination of kinetic reaction rate constants," IEEE Sensors Journal, vol. 9, no. 12, pp. 1697-1704, 2009.

[60] C. F. Carlborg, K. B. Gylfason, A. Kaźmierczak et al., "A packaged optical slot-waveguide ring resonator sensor array for multiplex label-free assays in labs-on-chips," Lab on a Chip, vol. 10, no. 3, pp. 281-290, 2010.

[61] C. J. Choi, A. R. Belobraydich, L. L. Chan, P. C. Mathias, and B. T. Cunningham, "Comparison of label-free biosensing in microplate, microfluidic, and spot-based affinity capture assays," Analytical Biochemistry, vol. 405, no. 1, pp. 1-10, 2010.

[62] C. Escobedo, A. G. Brolo, R. Gordon, and D. Sinton, "Flowthrough vs flow-over: analysis of transport and binding in nanohole array plasmonic biosensors," Analytical Chemistry, vol. 82, no. 24, pp. 10015-10020, 2010.

[63] J. N. Lee, C. Park, and G. M. Whitesides, "Solvent compatibility of poly(dimethylsiloxane)-based microfluidic devices," Analytical Chemistry, vol. 75, no. 23, pp. 6544-6554, 2003.

[64] G. M. Whitesides, "The origins and the future of microfluidics," Nature, vol. 442, no. 7101, pp. 368-373, 2006.

[65] D. Qin, Y. Xia, and G. M. Whitesides, "Soft lithography for micro- and nanoscale patterning," Nature Protocols, vol. 5, no. 3, pp. 491-502, 2010.

[66] S. Bhattacharya, A. Datta, J. M. Berg, and S. Gangopadhyay, "Studies on surface wettability of poly(dimethyl) siloxane (PDMS) and glass under oxygen-plasma treatment and correlation with bond strength," Journal of Microelectromechanical Systems, vol. 14, no. 3, pp. 590-597, 2005.

[67] S. Satyanarayana, R. N. Karnik, and A. Majumdar, "Stamp-andstick room-temperature bonding technique for microdevices," Journal of Microelectromechanical Systems, vol. 14, no. 2, pp. 392-399, 2005.

[68] S. H. Lee, N. C. Lindquist, N. J. Wittenberg, L. R. Jordan, and S.-H. Oh, "Real-time full-spectral imaging and affinity measurements from 50 microfluidic channels using nanohole surface plasmon resonance," Lab on a chip, vol. 12, no. 20, pp. 3882-3890, 2012.

[69] A. de Leebeeck, L. K. S. Kumar, V. de Lange, D. Sinton, R. Gordon, and A. G. Brolo, "On-chip surface-based detection with nanohole arrays," Analytical Chemistry, vol. 79, no. 11, pp. 4094-4100, 2007. 
[70] M. Huang, B. C. Galarreta, A. E. Cetin, and H. Altug, "Actively transporting virus like analytes with optofluidics for rapid and ultrasensitive biodetection," Lab on a Chip, vol. 13, no. 24, pp. 4841-4847, 2013.

[71] S.-H. Wu, K.-L. Lee, A. Chiou, X. Cheng, and P.-K. Wei, "Optofluidic platform for real-time monitoring of live cell secretory activities using Fano resonance in gold nanoslits," Small, vol. 9, no. 20, pp. 3532-3540, 2013.

[72] C. Escobedo, S. Vincent, A. I. K. Choudhury et al., "Integrated nanohole array surface plasmon resonance sensing device using a dual-wavelength source," Journal of Micromechanics and Microengineering, vol. 21, no. 11, Article ID 115001, 2011.

[73] H. K. Hunt and A. M. Armani, "Label-free biological and chemical sensors," Nanoscale, vol. 2, no. 9, pp. 1544-1559, 2010.

[74] H. K. Hunt and A. M. Armani, "Bioconjugation strategies for label-free optical microcavity sensors," IEEE Journal of Selected Topics in Quantum Electronics, vol. 20, no. 2, Article ID 6900213, 2014.

[75] O. Livnah, E. A. Bayer, M. Wilchek, and J. L. Sussman, "Three-dimensional structures of avidin and the avidin-biotin complex," Proceedings of the National Academy of Sciences of the United States of America, vol. 90, no. 11, pp. 5076-5080, 1993.

[76] A. Lesuffleur, H. Im, N. C. Lindquist, and S.-H. Oh, "Periodic nanohole arrays with shape-enhanced plasmon resonance as real-time biosensors," Applied Physics Letters, vol. 90, no. 24, Article ID 243110, 2007.

[77] C. Escobedo, Y.-W. Chou, M. Rahman et al., "Quantification of ovarian cancer markers with integrated microfluidic concentration gradient and imaging nanohole surface plasmon resonance," Analyst, vol. 138, no. 5, pp. 1450-1458, 2013.

[78] A. A. Yanik, A. E. Cetin, M. Huang et al., "Seeing protein monolayers with naked eye through plasmonic Fano resonances," Proceedings of the National Academy of Sciences of the United States of America, vol. 108, no. 29, pp. 11784-11789, 2011.

[79] A. A. Yanik, M. Huang, O. Kamohara et al., "An optofluidic nanoplasmonic biosensor for direct detection of live viruses from biological media," Nano Letters, vol. 10, no. 12, pp. $4962-$ 4969, 2010.

[80] Y. Sun and Y. Xia, "Increased sensitivity of surface plasmon resonance of gold nanoshells compared to that of gold solid colloids in response to environmental changes," Analytical Chemistry, vol. 74, no. 20, pp. 5297-5305, 2002.

[81] S.-W. Lee, K.-S. Lee, J. Ahn, J.-J. Lee, M.-G. Kim, and Y.-B. Shin, "Highly sensitive biosensing using arrays of plasmonic $\mathrm{Au}$ nanodisks realized by nanoimprint lithography," ACS Nano, vol. 5, no. 2, pp. 897-904, 2011.

[82] K.-S. Lee and M. A. El-Sayed, "Gold and silver nanoparticles in sensing and imaging: sensitivity of plasmon response to size, shape, and metal composition," Journal of Physical Chemistry B, vol. 110, no. 39, pp. 19220-19225, 2006.

[83] R. Bukasov, T. A. Ali, P. Nordlander, and J. S. ShumakerParry, "Probing the plasmonic near-field of gold nanocrescent antennas," ACS Nano, vol. 4, no. 11, pp. 6639-6650, 2010.

[84] E. M. Larsson, J. Alegret, M. Kall, and D. S. Sutherland, "Sensing characteristics of NIR localized surface plasmon resonances in gold nanorings for application as ultrasensitive biosensors," Nano Letters, vol. 7, no. 5, pp. 1256-1263, 2007.

[85] N. Verellen, P. van Dorpe, C. Huang et al., "Plasmon line shaping using nanocrosses for high sensitivity localized surface plasmon resonance sensing," Nano Letters, vol. 11, no. 2, pp. 391-397, 2011.
[86] H. Im, J. N. Sutherland, J. A. Maynard, and S. Oh, "Nanoholebased surface plasmon resonance instruments with improved spectral resolution quantify a broad range of antibody-ligand binding kinetics," Analytical Chemistry, vol. 84, pp. 1941-1947, 2012.

[87] T. W. Ebbesen, H. J. Lezec, H. F. Ghaemi, T. Thio, and P. A. Wolff, "Extraordinary optical transmission through sub-wavelength hole arrays," Nature, vol. 391, no. 6668, pp. 667-669, 1998.

[88] Q. Chen and D. R. S. Cumming, "High transmission and low color cross-talk plasmonic color filters using triangular-lattice hole arrays in aluminum films," Optics Express, vol. 18, no. 13, pp. 14056-14062, 2010.

[89] J. Henzie, M. H. Lee, and T. W. Odom, "Multiscale patterning of plasmonic metamaterials," Nature Nanotechnology, vol. 2, no. 9, pp. 549-554, 2007.

[90] P. Nagpal, N. C. Lindquist, S.-H. Oh, and D. J. Norris, "Ultrasmooth patterned metals for plasmonics and metamaterials," Science, vol. 325, no. 5940, pp. 594-597, 2009.

[91] S. Koynov, M. S. Brandt, and M. Stutzmann, "Black nonreflecting silicon surfaces for solar cells," Applied Physics Letters, vol. 88, no. 20, Article ID 203107, 2006.

[92] S. Kalem, P. Werner, Ö. Arthursson et al., "Black silicon with high density and high aspect ratio nanowhiskers," Nanotechnology, vol. 22, no. 23, Article ID 235307, 2011.

[93] A. Lesuffleur, H. Im, N. C. Lindquist, and S. Oh, "Periodic nanohole arrays with shape-enhanced plasmon resonance as real-time biosensors," Applied Physics Letters, vol. 90, no. 24, Article ID 243110, 2007. 

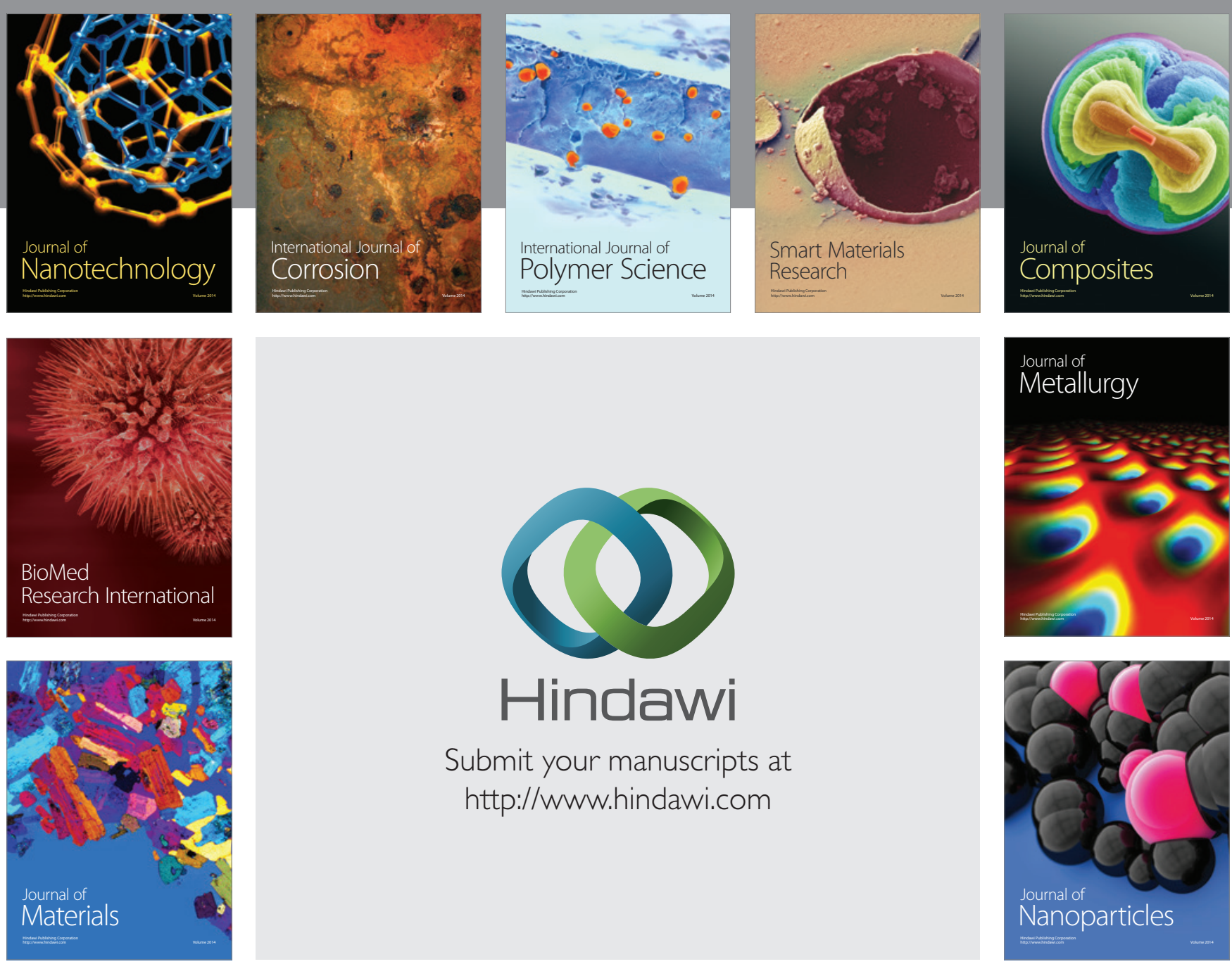

Submit your manuscripts at http://www.hindawi.com
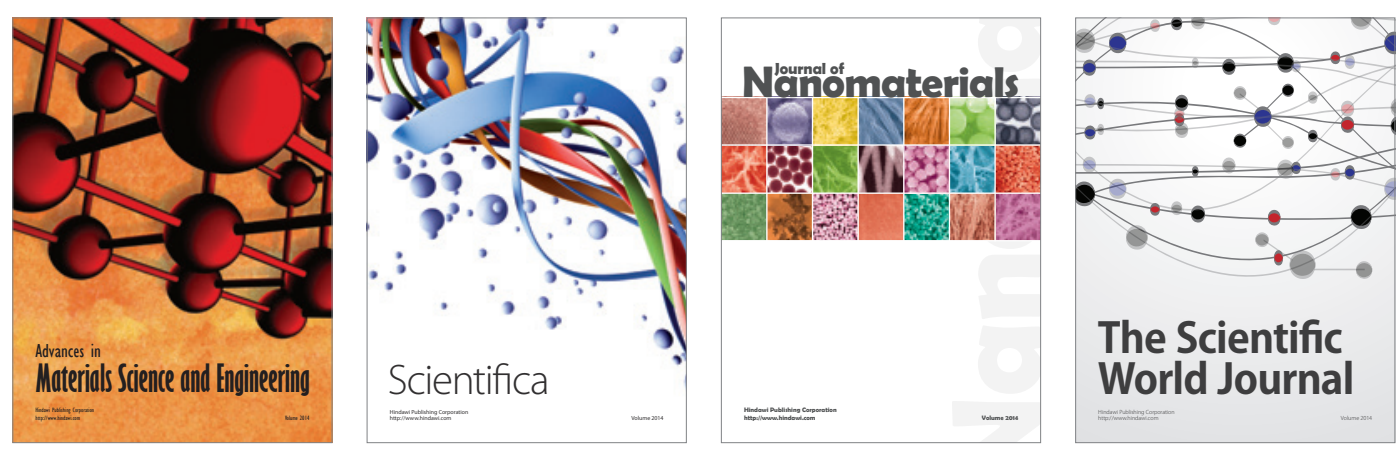

\section{The Scientific World Journal}
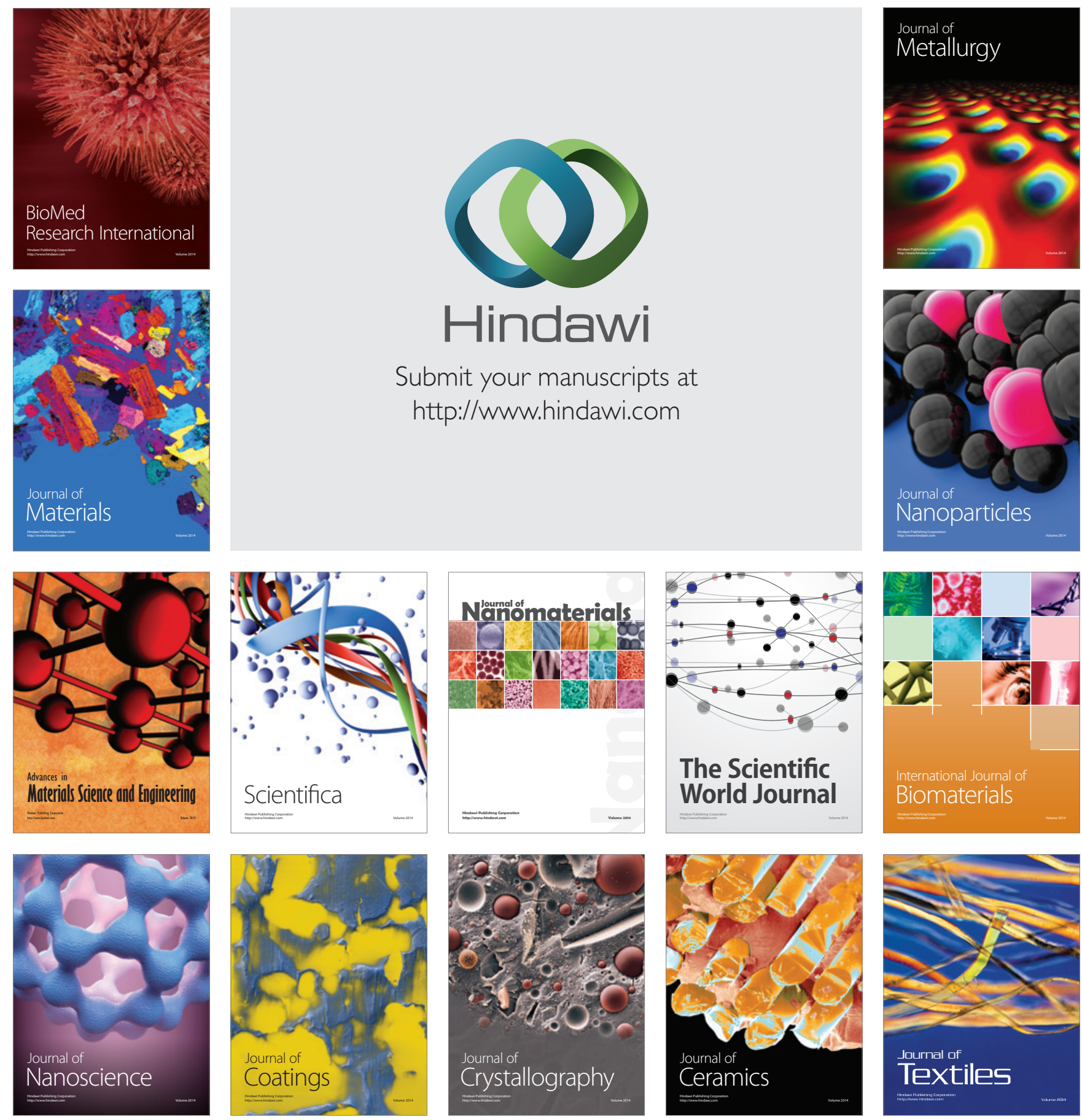